\title{
PRICING BONDS AND CDS IN THE MODEL WITH RATING MIGRATION INDUCED BY A COX PROCESS
}

\author{
JACEK JAKUBOWSKI \\ Institute of Mathematics, University of Warsaw \\ Banacha 2, 02-097 Warszawa, Poland \\ E-mail: jakub@mimuw.edu.pl \\ and \\ Faculty of Mathematics and Information Science \\ Warsaw University of Technology \\ MARIUSZ NIEWĘGŁOWSKI \\ Faculty of Mathematics and Information Science, Warsaw University of Technology \\ Plac Politechniki 1, 00-661 Warszawa, Poland \\ E-mail: M.Nieweglowski@mini.pw.edu.pl
}

\begin{abstract}
We investigate the properties of a rating migration process assuming that it is given by subordination of a discrete time Markov chain and a Cox process. The problem of pricing of defaultable bonds with fractional recovery of par value with rating migration and credit default swaps is considered. As an example of applications of our results, we give an explicit solution to the pricing problem in a model with short rate and intensity processes given by the solution of a two-dimensional Ornstein-Uhlenbeck equation with a Lévy noise.
\end{abstract}

1. Introduction. Jarrow, Lando and Turnbull in the seminal paper [15] introduced Markov chains to model evolution in time of credit ratings. Lando [16] has extended work of Jarrow et al. by introducing the rating migration process which follows the so called conditional Markov chain (see Bielecki and Rutkowski [5] for the precise definition of conditional Markov property). The conditional generator of the credit rating process follows a matrix-valued stochastic process, under construction provided by Lando [16] and Bielecki and Rutkowski [5]. Lando [16] has considered the problem of providing

2000 Mathematics Subject Classification: Primary 62P05; Secondary 60J27, 91B70.

Key words and phrases: credit risk, rating migration, defaultable bonds, credit default swap, Cox processes, conditional Markov chains.

Research supported by KBN grant "Stochastic evolution equations driven by Lévy noise". The paper is in final form and no version of it will be published elsewhere. 
explicit formulae for some credit derivatives connected with ratings. He has shown that under the assumption that the generator has eigenvectors constant in time, it is possible to solve the conditional Kolmogorov equation and obtain explicit formulae for the bond prices and rating-dependent payoffs. Many papers are concerned with pricing of credit derivatives with rating migrations, for example Acharya, Das and Sundaram [1], Das and Tufano [6]. For the extension of the HJM methodology to the case of defaultable bonds with rating migrations see Bielecki and Rutkowski [3], and for models with Lévy noise see Eberlein and Özkan [8]. Some recent papers consider infinite-dimensional noise in HJM type models: see Schmidt [20], Jakubowski and Zabczyk [14] and Jakubowski, Niewęgłowski and Zabczyk [13]. It is also worth to mention recent papers by Bielecki et al. [2] and Hurd and Kuznetsov [11], [12] concerning the valuation of basket credit derivatives in a rating migration environment.

In this paper we consider the problem of pricing defaultable bonds and credit default swaps with rating migration. First, we derive formulae for conditional expectations involving the rating migration process. We focus on the case of payoffs which depend on the time of default and on the state of the rating process before default (pre-default rating). Such payoffs appear naturally when one considers defaultable bonds with recovery payment dependent on a pre-default rating, and also in the case of credit default swaps connected with such bonds. In the third section we consider a rating migration process $C$ which is constructed by superposition (subordination) of a discrete time-homogeneous Markov chain $\bar{C}$ with finite state space with one absorbing state and the remaining states transient, and a Cox process $N$ (independent of the Markov chain $\bar{C}$ ), i.e. $C_{t}:=\bar{C}_{N_{t}}$. The default is defined as the time at which the rating migration process enters the absorbing state. We prove that, under this construction, the rating migration process is a conditionally Markov process and also that the so called $(\mathrm{H})$ hypothesis, known in credit risk theory, holds. We present the form of the joint conditional distribution of the default time and the pre-default state of the rating migration process in terms of the exponential of some matrix. Then, we give an explicit formula for it in terms of the Jordan decomposition of the transition matrix. In the fourth section, we use all these results to solve the problem of pricing of defaultable bonds with fractional recovery of par value and pricing of credit default swaps (CDS). As an example of applications of our results we present a model for which we find an explicit solution to the pricing problem. In this example, short rate and intensity processes are given as the solution of a two-dimensional Ornstein-Uhlenbeck equation with a Lévy noise. In the appendix, we prove useful lemmas on the credit rating process induced by a Cox process, which are of independent interest. We give the proof of Lemma 2 which states a general fact on conditional independence and quote, for the convenience of the reader, facts from matrix algebra used in the previous sections.

2. Useful conditional expectations for processes of credit migrations. We consider an arbitrage-free market on which defaultable instruments are also traded. All processes are defined on a complete probability space $(\Omega, \mathcal{F}, \mathbf{P})$. A credit rating migration process $C$ of a defaultable instrument (e.g. bond) is a càdlàg process which takes values 
in the set of rating classes $\mathcal{K}=\{1, \ldots, K\}$, where the state $i=1$ represents the highest rank, $i=K-1$ the lowest rank and the state $i=K$ the bankruptcy, so the state $K$ corresponds to the default event. Therefore,

$$
\tau:=\inf \left\{t>0: C_{t}=K\right\}
$$

is the default time. Here we preserve the interpretation of rating classes, i.e. the fact the higher rated bonds are more expensive than lower rated. In this section we present some general formulae for conditional expectations useful in the pricing of defaultable claims. We will denote by $\mathbb{F}$ the reference filtration corresponding to observation of the market without credit rating, i.e. a filtration corresponding to the interest rate risk and other market factors that drive the credit risk. As usual, $\mathbb{F}^{X}$ denotes the filtration generated by the process $X$, i.e. $\mathcal{F}_{t}^{X}=\sigma\left(X_{u}: u \leq t\right)$.

All results in this paper will be proved under the following assumptions:

Assumption 1. For every $i \in \mathcal{K}$ and any $u \geq t \geq 0$ we have

$$
\mathbf{P}\left(C_{u}=i \mid \mathcal{F}_{\infty} \vee \mathcal{F}_{t}^{C}\right)=\mathbf{P}\left(C_{u}=i \mid \mathcal{F}_{\infty} \vee \sigma\left(C_{t}\right)\right) .
$$

Assumption 2. For every $\mathcal{F}_{\infty}$ measurable integrable random variable $Y$ we have

$$
\mathbf{E}\left(Y \mid \mathcal{F}_{t} \vee \mathcal{F}_{t}^{C}\right)=\mathbf{E}\left(Y \mid \mathcal{F}_{t}\right) \quad \forall t \in \mathbf{R}_{+} .
$$

Assumption 3. For every $i \in \mathcal{K}$ and for every $t>0$ we have

$$
\mathbf{P}\left(C_{t}=i \mid \mathcal{F}_{t}\right)>0 \text {. }
$$

In the next section we give an example of a rating process for which these assumptions are satisfied.

REMARK 1. a) It is also worth to note that Assumption 1 is redundant if $K=2$ (so we have only two states: default and non-default). This follows from rephrasing of Lemma 5.1.4, p. 146 in Bielecki, Rutkowski [4]. Under the assumption $\mathbf{P}\left(C_{t}=1 \mid \mathcal{F}_{\infty}\right)>0$, this lemma implies that for $u \geq t$,

$$
\mathbf{P}\left(C_{u}=1 \mid \mathcal{F}_{\infty} \vee \mathcal{F}_{t}^{C}\right)=\mathbf{1}_{\left\{C_{t}=1\right\}} \frac{\mathbf{P}\left(C_{u}=1 \mid \mathcal{F}_{\infty}\right)}{\mathbf{P}\left(C_{t}=1 \mid \mathcal{F}_{\infty}\right)} .
$$

Since $\left\{C_{u}=1\right\} \subseteq\left\{C_{t}=1\right\}$, we have

$$
\begin{aligned}
\mathbf{P}\left(C_{u}=1 \mid \mathcal{F}_{\infty} \vee \mathcal{F}_{t}^{C}\right) & =\mathbf{1}_{\left\{C_{t}=1\right\}} \frac{\mathbf{P}\left(C_{u}=1, C_{t}=1 \mid \mathcal{F}_{\infty}\right)}{\mathbf{P}\left(C_{t}=1 \mid \mathcal{F}_{\infty}\right)} \\
& =\mathbf{1}_{\left\{C_{t}=1\right\}} \mathbf{P}\left(C_{u}=1 \mid \mathcal{F}_{\infty} \vee \sigma\left(C_{t}\right)\right),
\end{aligned}
$$

where in the last equality we use the same arguments as in the proof of Lemma 3 below.

b) If we fix an event $A$ belonging to $\mathcal{F}_{\infty}$, then by Assumption $1, C$ is a Markov process (usually time inhomogeneous) conditionally on that event $A$. This assumption is in the spirit of the theory of doubly stochastic Poisson processes, so we could say that processes which satisfy this property are doubly stochastic Markov chains opposite to the conditional Markov chains (see Definition 11.2.1 in Bielecki and Rutkowski [5]). Assumption 1 is satisfied for the construction presented in the third section of this paper, and also for the construction in Bielecki and Rutkowski [5] and Lando [16]. 
c) Assumption 2 for $K=2$ is well known in the default risk literature and there are many different equivalent formulations of this assumption (see Bielecki and Rutkowski [5] section 6.1.1).

d) Assumption 3 means that each state is attainable with positive probability. In particular, this means that for any small $t>0$ the process $C$ can reach the state $K$ (i.e. the default state) with positive probability, although this probability may be very small.

e) Assumption 3 also implies that $\mathcal{F}_{t}^{C} \subsetneq \mathcal{F}_{t}$.

It is easily seen that Assumption 1 can be expressed in a different way, namely

Proposition 1. The following conditions are equivalent:

a) Assumption 1 .

b) For every $t \geq 0$ and every bounded random variable $X$ which is $\mathcal{F}^{C, t}:=\sigma\left\{C_{u}: u \geq t\right\}$ measurable we have

$$
\mathbf{E}\left(X \mid \mathcal{F}_{\infty} \vee \mathcal{F}_{t}^{C}\right)=\mathbf{E}\left(X \mid \mathcal{F}_{\infty} \vee \sigma\left(C_{t}\right)\right)
$$

In the next lemma we give an equivalent formulation of Assumption 2.

Lemma 2. Assumption 2 is equivalent to any of the two following conditions:

i) For each $t \in \mathbf{R}_{+}$, the $\sigma$-fields $\mathcal{F}_{\infty}$ and $\mathcal{F}_{t}^{C}$ are conditionally independent given $\mathcal{F}_{t}$.

ii) For each $n \in \mathbf{N}$ and any sequences $0 \leq u_{1}<\ldots<u_{n} \leq t,\left(i_{1}, \ldots, i_{n}\right) \in \mathcal{K}^{n}$ the following equality holds

$$
\mathbf{P}\left(C_{u_{1}}=i_{1}, \ldots, C_{u_{n}}=i_{n} \mid \mathcal{F}_{t}\right)=\mathbf{P}\left(C_{u_{1}}=i_{1}, \ldots, C_{u_{n}}=i_{n} \mid \mathcal{F}_{\infty}\right) .
$$

The proof of this simple, but very useful lemma will be given in the appendix.

Now we prove a general fact on conditional expectation. It is a key lemma in later calculations.

Lemma 3. Let $X$ be an integrable random variable on $(\Omega, \mathcal{M}, \mathbf{P})$ and let $Y$ be a random variable which takes a finite number of values $y_{1}, \ldots, y_{K}$. For an arbitrary $\sigma$-field $\mathcal{G} \subset \mathcal{M}$ such that

$$
\mathbf{P}\left(Y=y_{i} \mid \mathcal{G}\right)>0 \quad \text { for } \quad i=1, \ldots, K
$$

we have

$$
\mathbf{E}(X \mid \mathcal{G} \vee \sigma(Y))=\sum_{i=1}^{K} \mathbf{1}_{\left\{Y=y_{i}\right\}} \frac{\mathbf{E}\left(X \mathbf{1}_{\left\{Y=y_{i}\right\}} \mid \mathcal{G}\right)}{\mathbf{P}\left(Y=y_{i} \mid \mathcal{G}\right)} .
$$

Proof. It is sufficient to prove that

$$
\mathbf{E}(X \mid \mathcal{G} \vee \sigma(Y)) \mathbf{1}_{\left\{Y=y_{i}\right\}}=\mathbf{1}_{\left\{Y=y_{i}\right\}} \frac{\mathbf{E}\left(X \mathbf{1}_{\left\{Y=y_{i}\right\}} \mid \mathcal{G}\right)}{\mathbf{P}\left(Y=y_{i} \mid \mathcal{G}\right)},
$$

so we have to show that for every $A \in \mathcal{G} \vee \sigma(Y)$,

$$
\int_{A} X \mathbf{1}_{\left\{Y=y_{i}\right\}} \mathbf{P}\left(Y=y_{i} \mid \mathcal{G}\right) d \mathbf{P}=\int_{A} \mathbf{1}_{\left\{Y=y_{i}\right\}} \mathbf{E}\left(X \mathbf{1}_{\left\{Y=y_{i}\right\}} \mid \mathcal{G}\right) d \mathbf{P} .
$$

The class $\mathcal{L}$ of $\mathcal{M}$-measurable sets satisfying (5) constitutes a $\lambda$-system. Let $\mathcal{P}$ denote the class of sets of the following form

$$
\mathcal{P}=\left\{A \in \mathcal{M}: \exists B \in \mathcal{G}, \exists y_{j} \in\left\{y_{1}, \ldots, y_{K}\right\} \text { such that } A=B \cap\left\{Y=y_{j}\right\}\right\} .
$$


$\mathcal{P}$ is a $\pi$-system. Moreover the $\sigma$-field generated by $\mathcal{P}$ is equal to $\mathcal{G} \vee \sigma(Y)$. Therefore, by the lemma on $\pi-\lambda$ systems, to prove (5) for sets from $\mathcal{G} \vee \sigma(Y)$ it is enough to prove it for sets from $\mathcal{P}$. To end the proof it is enough to show (5) for $y_{j}=y_{i}$, since it is obvious for $y_{j} \neq y_{i}$ :

$$
\begin{aligned}
L H S & =\int_{B \cap\left\{Y=y_{i}\right\}} X \mathbf{1}_{\left\{Y=y_{i}\right\}} \mathbf{P}\left(Y=y_{i} \mid \mathcal{G}\right) d \mathbf{P} \\
& =\int_{B} \mathbf{E}\left(X \mathbf{1}_{\left\{Y=y_{i}\right\}} \mathbf{E}\left(\mathbf{1}_{\left\{Y=y_{i}\right\}} \mid \mathcal{G}\right) \mid \mathcal{G}\right) d \mathbf{P} \\
& =\int_{B} \mathbf{E}\left(X \mathbf{1}_{\left\{Y=y_{i}\right\}} \mid \mathcal{G}\right) \mathbf{E}\left(\mathbf{1}_{\left\{Y=y_{i}\right\}} \mid \mathcal{G}\right) d \mathbf{P} \\
& =\int_{B} \mathbf{E}\left(\mathbf{1}_{\left\{Y=y_{i}\right\}} \mathbf{E}\left(X \mathbf{1}_{\left\{Y=y_{i}\right\}} \mid \mathcal{G}\right) \mid \mathcal{G}\right) d \mathbf{P} \\
& =\int_{B} \mathbf{1}_{\left\{Y=y_{i}\right\}} \mathbf{E}\left(X \mathbf{1}_{\left\{Y=y_{i}\right\}} \mid \mathcal{G}\right) d \mathbf{P} \\
& =\int_{B \cap\left\{Y=y_{i}\right\}} \mathbf{1}_{\left\{Y=y_{i}\right\}} \mathbf{E}\left(X \mathbf{1}_{\left\{Y=y_{i}\right\}} \mid \mathcal{G}\right) d \mathbf{P}=R H S .
\end{aligned}
$$

REMARK 2. By Assumption 3

$$
\tilde{p}_{i, j}(t, u):=\frac{\mathbf{P}\left(C_{u}=j, C_{t}=i \mid \mathcal{F}_{\infty}\right)}{\mathbf{P}\left(C_{t}=i \mid \mathcal{F}_{\infty}\right)}
$$

is a well defined $\mathcal{F}_{\infty}$ measurable random variable. Using Lemma 3 we obtain

$$
\mathbf{P}\left(C_{u}=j \mid \mathcal{F}_{\infty} \vee \mathcal{F}_{t}^{C}\right) \mathbf{1}_{\left\{C_{t}=i\right\}}=\tilde{p}_{i, j}(t, u) \mathbf{1}_{\left\{C_{t}=i\right\}}
$$

Now we derive some conditional expectations useful in pricing of defaultable bonds with credit rating migration and their derivatives:

TheOREM 4. Let $X$ be an $\mathcal{F}_{\infty}$-measurable integrable random variable. Then

a) for every $0 \leq t \leq u$ and every $j \in \mathcal{K}$

$$
\mathbf{E}\left(X \mathbf{1}_{\left\{C_{u}=j\right\}} \mid \mathcal{F}_{t} \vee \mathcal{F}_{t}^{C}\right)=\sum_{i=1}^{K} \mathbf{1}_{\left\{C_{t}=i\right\}} \mathbf{E}\left(X \tilde{p}_{i, j}(t, u) \mid \mathcal{F}_{t}\right)
$$

b) for every $0 \leq t \leq u$ and $j \in \mathcal{K} \backslash\{K\}$

$$
\mathbf{E}\left(X \mathbf{1}_{\left\{t<\tau \leq u, C_{\tau-}=j\right\}} \mid \mathcal{F}_{t} \vee \mathcal{F}_{t}^{C}\right)=\sum_{i=1}^{K-1} \mathbf{1}_{\left\{C_{t}=i\right\}} \mathbf{E}\left(X G_{t, i, j}(u) \mid \mathcal{F}_{t}\right),
$$

where

$$
G_{t, i, j}(u):=\frac{\mathbf{P}\left(t<\tau \leq u, C_{\tau-}=j, C_{t}=i \mid \mathcal{F}_{\infty}\right)}{\mathbf{P}\left(C_{t}=i \mid \mathcal{F}_{\infty}\right)}
$$

for $i, j \in \mathcal{K} \backslash K$ and $u \geq t$.

Proof. a) It is enough to show equality (7) on the set $\left\{C_{t}=i\right\}$. Using, successively, the chain rule for conditional expectation, Assumption 1, Lemma 3, Assumption 2 we have 


$$
\begin{aligned}
& \mathbf{E}\left(X \mathbf{1}_{\left\{C_{u}=j\right\}} \mid \mathcal{F}_{t} \vee \mathcal{F}_{t}^{C}\right) \mathbf{1}_{\left\{C_{t}=i\right\}}=\mathbf{E}\left(X \mathbf{E}\left(\mathbf{1}_{\left\{C_{u}=j\right\}} \mid \mathcal{F}_{\infty} \vee \mathcal{F}_{t}^{C}\right) \mathbf{1}_{\left\{C_{t}=i\right\}} \mid \mathcal{F}_{t} \vee \mathcal{F}_{t}^{C}\right) \\
& =\mathbf{E}\left(X \mathbf{E}\left(\mathbf{1}_{\left\{C_{u}=j\right\}} \mid \mathcal{F}_{\infty} \vee \sigma\left(C_{t}\right)\right) \mathbf{1}_{\left\{C_{t}=i\right\}} \mid \mathcal{F}_{t} \vee \mathcal{F}_{t}^{C}\right) \\
& =\mathbf{E}\left(X \frac{\mathbf{E}\left(\mathbf{1}_{\left\{C_{u}=j, C_{t}=i\right\}} \mid \mathcal{F}_{\infty}\right)}{\mathbf{P}\left(C_{t}=i \mid \mathcal{F}_{\infty}\right)} \mathbf{1}_{\left\{C_{t}=i\right\}} \mid \mathcal{F}_{t} \vee \mathcal{F}_{t}^{C}\right) \\
& =\mathbf{1}_{\left\{C_{t}=i\right\}} \mathbf{E}\left(X \tilde{p}_{i, j}(t, u) \mid \mathcal{F}_{t} \vee \mathcal{F}_{t}^{C}\right)=\mathbf{1}_{\left\{C_{t}=i\right\}} \mathbf{E}\left(X \tilde{p}_{i, j}(t, u) \mid \mathcal{F}_{t}\right) .
\end{aligned}
$$

b) The proof is similar to the proof of part a). Using (1) with $X=\mathbf{1}_{A}$ and

$$
A:=\left\{t<\tau \leq u, C_{\tau-}=j\right\} \in \mathcal{F}^{C, t}
$$

we obtain

$$
\mathbf{P}\left(t<\tau \leq u, C_{\tau-}=j \mid \mathcal{F}_{\infty} \vee \mathcal{F}_{t}^{C}\right)=\mathbf{P}\left(t<\tau \leq u, C_{\tau-}=j \mid \mathcal{F}_{\infty} \vee \sigma\left(C_{t}\right)\right)
$$

Hence

$$
\begin{aligned}
\mathbf{E}\left(X \mathbf{1}_{A} \mid \mathcal{F}_{t} \vee \mathcal{F}_{t}^{C}\right) \mathbf{1}_{\left\{C_{t}=i\right\}} & =\mathbf{E}\left(X \mathbf{E}\left(\mathbf{1}_{A} \mid \mathcal{F}_{\infty} \vee \mathcal{F}_{t}^{C}\right) \mathbf{1}_{\left\{C_{t}=i\right\}} \mid \mathcal{F}_{t} \vee \mathcal{F}_{t}^{C}\right) \\
& =\mathbf{E}\left(X \mathbf{E}\left(\mathbf{1}_{A} \mid \mathcal{F}_{\infty} \vee \sigma\left(C_{t}\right)\right) \mathbf{1}_{\left\{C_{t}=i\right\}} \mid \mathcal{F}_{t} \vee \mathcal{F}_{t}^{C}\right) \\
& =\mathbf{E}\left(X \frac{\mathbf{E}\left(\mathbf{1}_{A \cap\left\{C_{t}=i\right\}} \mid \mathcal{F}_{\infty}\right)}{\mathbf{P}\left(C_{t}=i \mid \mathcal{F}_{\infty}\right)} \mathbf{1}_{\left\{C_{t}=i\right\}} \mid \mathcal{F}_{t} \vee \mathcal{F}_{t}^{C}\right) \\
& =\mathbf{1}_{\left\{C_{t}=i\right\}} \mathbf{E}\left(X \frac{\mathbf{P}\left(A \cap\left\{C_{t}=i\right\} \mid \mathcal{F}_{\infty}\right)}{\mathbf{P}\left(C_{t}=i \mid \mathcal{F}_{\infty}\right)} \mid \mathcal{F}_{t}\right) \\
& =\mathbf{1}_{\left\{C_{t}=i\right\}} \mathbf{E}\left(X G_{t, i, j}(u) \mid \mathcal{F}_{t}\right)
\end{aligned}
$$

where the second equality is a consequence of property (10), the third follows from Lemma 3 and the fourth equality follows from Assumption 2 applied to the integrable random variable $Y=\mathbf{E}\left(X \mathbf{1}_{A \cap\left\{C_{t}=i\right\}} \mid \mathcal{F}_{\infty}\right)$.

COROLlary 5. For every bounded $\mathcal{F}_{\infty}$-measurable random variable $X$ and for any $u \geq t$

$$
\mathbf{E}\left(X \mathbf{1}_{\{\tau>u\}} \mid \mathcal{F}_{t} \vee \mathcal{F}_{t}^{C}\right)=\sum_{i=1}^{K} \mathbf{1}_{\left\{C_{t}=i\right\}} \mathbf{E}\left(X \sum_{j=1}^{K-1} \tilde{p}_{i, j}(t, u) \mid \mathcal{F}_{t}\right) .
$$

Proof. Since $\{\tau>u\}=\left\{C_{u} \neq K\right\}$, we obtain formula (11) by applying (7).

TheOREM 6. Let $Z$ be a bounded, $\mathbb{F}$-predictable stochastic process. Then we have:

a) for every $0 \leq t \leq u$

$$
\mathbf{E}\left(Z_{\tau} \mathbf{1}_{\{t<\tau \leq u\}} \mid \mathcal{F}_{t} \vee \mathcal{F}_{t}^{C}\right)=\sum_{i=1}^{K-1} \mathbf{1}_{\left\{C_{t}=i\right\}} \mathbf{E}\left(\int_{t}^{u} Z_{v} d F_{t, i}(v) \mid \mathcal{F}_{t}\right),
$$

where

$$
F_{i, t}(v):=\frac{\mathbf{P}\left(\tau \leq v, C_{t}=i \mid \mathcal{F}_{\infty}\right)}{\mathbf{P}\left(C_{t}=i \mid \mathcal{F}_{\infty}\right)}
$$

for every $0 \leq t \leq v$ and for each $i \in \mathcal{K} \backslash K$,

b) for every $0 \leq t \leq u$

$$
\mathbf{E}\left(Z_{\tau} \mathbf{1}_{\left\{t<\tau \leq u, C_{\tau-}=j\right\}} \mid \mathcal{F}_{t} \vee \mathcal{F}_{t}^{C}\right)=\sum_{i=1}^{K-1} \mathbf{1}_{\left\{C_{t}=i\right\}} \mathbf{E}\left(\int_{t}^{u} Z_{v} d G_{t, i, j}(v) \mid \mathcal{F}_{t}\right) .
$$


REMARK 3. From condition ii) in Lemma 2 it follows that the process $F_{i, t}$, for fixed $i, t$, is nondecreasing, so the integral under conditional expectation in (12) should be understood as the Lebesgue-Stieltjes integral. The same concerns $G_{t, i, j}$.

REMARK 4. Notice that

$$
F_{i, t}(v):=\frac{\mathbf{P}\left(\tau \leq v, C_{t}=i \mid \mathcal{F}_{\infty}\right)}{\mathbf{P}\left(C_{t}=i \mid \mathcal{F}_{\infty}\right)}=\frac{\mathbf{P}\left(C_{v}=K, C_{t}=i \mid \mathcal{F}_{\infty}\right)}{\mathbf{P}\left(C_{t}=i \mid \mathcal{F}_{\infty}\right)}=\tilde{p}_{i, K}(t, v) .
$$

Proof of theorem. Let us consider an $\mathbb{F}$-predictable simple process, i.e. a process of the following form

$$
Z_{s}=\sum_{k=1}^{n} \xi_{k} \mathbf{1}_{\left\{t_{k}<s \leq t_{k+1}\right\}}
$$

where $\xi_{k}$ is a bounded $\mathcal{F}_{t_{k}}$-measurable random variable.

a) Note that $\left\{\tau \leq v, C_{t}=i\right\} \in \mathcal{F}^{C, t}$ for $v \geq t$. Hence and by Proposition 1 it follows that

$$
\begin{aligned}
\mathbf{E}\left(\xi_{k} \mathbf{1}_{\{\tau \leq v\}} \mid \mathcal{F}_{t} \vee \mathcal{F}_{t}^{C}\right) \mathbf{1}_{\left\{C_{t}=i\right\}} & =\mathbf{E}\left(\xi_{k} \mathbf{E}\left(\mathbf{1}_{\left\{\tau \leq v, C_{t}=i\right\}} \mid \mathcal{F}_{\infty} \vee \mathcal{F}_{t}^{C}\right) \mathbf{1}_{\left\{C_{t}=i\right\}} \mid \mathcal{F}_{t} \vee \mathcal{F}_{t}^{C}\right) \\
& =\mathbf{E}\left(\xi_{k} \mathbf{E}\left(\mathbf{1}_{\left\{\tau \leq v, C_{t}=i\right\}} \mid \mathcal{F}_{\infty} \vee \sigma\left(C_{t}\right)\right) \mathbf{1}_{\left\{C_{t}=i\right\}} \mid \mathcal{F}_{t} \vee \mathcal{F}_{t}^{C}\right) \\
& =\mathbf{E}\left(\xi_{k} \frac{\mathbf{P}\left(\tau \leq v, C_{t}=i \mid \mathcal{F}_{\infty}\right)}{\mathbf{P}\left(C_{t}=i \mid \mathcal{F}_{\infty}\right)} \mathbf{1}_{\left\{C_{t}=i\right\}} \mid \mathcal{F}_{t} \vee \mathcal{F}_{t}^{C}\right) \\
& =\mathbf{E}\left(\xi_{k} F_{t, i}(v) \mid \mathcal{F}_{t} \vee \mathcal{F}_{t}^{C}\right) \mathbf{1}_{\left\{C_{t}=i\right\}} \\
& =\mathbf{E}\left(\xi_{k} F_{t, i}(v) \mid \mathcal{F}_{t}\right) \mathbf{1}_{\left\{C_{t}=i\right\}},
\end{aligned}
$$

where in the third equality we have used Lemma 3 and in the fifth equality Assumption 2. Using (16) for $v=t_{k}$ and $v=t_{k+1}$ we obtain

$$
\begin{aligned}
& \mathbf{E}\left(\xi_{k} \mathbf{1}_{\left\{t_{k}<\tau \leq t_{k+1}\right\}} \mid \mathcal{F}_{t} \vee \mathcal{F}_{t}^{C}\right) \mathbf{1}_{\left\{C_{t}=i\right\}} \\
& =\mathbf{1}_{\left\{C_{t}=i\right\}} \mathbf{E}\left(\xi_{k}\left(F_{t, i}\left(t_{k+1}\right)-F_{t, i}\left(t_{k}\right)\right) \mid \mathcal{F}_{t}\right)=\mathbf{1}_{\left\{C_{t}=i\right\}} \mathbf{E}\left(\int_{t_{k}}^{t_{k+1}} Z_{v} d F_{t, i}(v) \mid \mathcal{F}_{t}\right)
\end{aligned}
$$

Since $Z_{\tau}$ for $Z$ given by (15) is equal to

$$
Z_{\tau}=\sum_{k=1}^{n} \xi_{k} \mathbf{1}_{\left\{t_{k}<\tau \leq t_{k+1}\right\}}
$$

(17) gives formula (12) for an $\mathbb{F}$-predictable simple processes. For a general bounded $\mathbb{F}$ predictable process the assertion follows by the monotone class theorem (see e.g. Protter [19] page 7).

b) The proof goes in the same manner as in point a). It is enough to prove (14) for an $\mathbb{F}$-predictable process $Z$ of the form (15). Applying the monotone class theorem we obtain the general case. Now, we briefly sketch the main steps of the proof.

For every $v \geq t$ formula (8) implies

$$
\mathbf{E}\left(\xi_{k} \mathbf{1}_{\left\{t<\tau \leq v, C_{\tau-}=j\right\}} \mid \mathcal{F}_{t} \vee \mathcal{F}_{t}^{C}\right) \mathbf{1}_{\left\{C_{t}=i\right\}}=\mathbf{1}_{\left\{C_{t}=i\right\}} \mathbf{E}\left(\xi_{k} G_{t, i, j}(v) \mid \mathcal{F}_{t}\right) .
$$

To complete the proof for a simple $\mathbb{F}$-predictable process $Z$ it is enough to notice that for $t_{k+1}>t_{k} \geq t$ we have

$$
\mathbf{E}\left(\xi_{k} \mathbf{1}_{\left\{t_{k}<\tau \leq t_{k+1}, C_{\tau-}=j, C_{t}=i\right\}} \mid \mathcal{F}_{t} \vee \mathcal{F}_{t}^{C}\right)=\mathbf{1}_{\left\{C_{t}=i\right\}} \mathbf{E}\left(\xi_{k}\left(G_{t, i, j}\left(t_{k+1}\right)-G_{t, i, j}\left(t_{k}\right)\right) \mid \mathcal{F}_{t}\right),
$$

which follows from equality (18) applied to $v=t_{k+1}$ and $v=t_{k}$. 
3. Model of rating migration induced by a Cox process. Let $\mathbb{F}$ be a given reference filtration which contains all market information apart from the information about rating migration. We construct a rating migration process using the following objects:

1. A discrete time homogeneous Markov chain $\left(\bar{C}_{k}\right)_{k \geq 0}$, independent of $\mathcal{F}_{\infty}$, with values in the set $\mathcal{K}=\{1, \ldots, K\}$. We assume that $\bar{C}$ has one absorbing state $K$ and the remaining states are transient. So the transition matrix, denoted by $P$, is of the following form

$$
P=\left[\begin{array}{ccccc}
p_{1,1} & p_{1,2} & p_{1,3} & \ldots & p_{1, K} \\
p_{2,1} & p_{2,2} & p_{2,3} & \ldots & p_{2, K} \\
p_{3,1} & p_{3,2} & p_{3,3} & \ldots & p_{3, K} \\
\vdots & \vdots & \vdots & \ddots & \vdots \\
p_{K-1,1} & p_{K-1,2} & p_{K-1,2} & \ldots & p_{K-1, K} \\
0 & 0 & 0 & 0 & 1
\end{array}\right]
$$

2. A Cox process $\left(N_{t}\right)_{t \geq 0}$ with an $\mathbb{F}$-adapted intensity $\left(\lambda_{t}\right)_{t \geq 0}$.

We recall here the definition of Cox processes (conditional Poisson processes, see e.g. [4]).

Definition 1. A stochastic process $\left(N_{t}\right)_{t \geq 0}$ is called a Cox process if there exists a nonnegative $\mathbb{F}$-adapted stochastic process $\left(\lambda_{t}\right)_{t \geq 0}$ which satisfies

$$
\mathbf{P}\left(\int_{0}^{t} \lambda_{u} d u<+\infty ; \quad \forall t>0\right)=1
$$

and

$$
\mathbf{P}\left(\int_{0}^{\infty} \lambda_{u} d u=\infty\right)=1,
$$

and such that the process $\left(N_{t}\right)_{t \geq 0}$ conditionally on realization of the intensity, i.e. conditionally on the $\sigma$-field $\mathcal{F}_{\infty}^{\lambda}$, is a time inhomogeneous Poisson process with the intensity $\left(\lambda_{t}\right)_{t \geq 0}$. This means that for all $0 \leq s<t<\infty$ we have

$$
\mathbf{E}\left(e^{i u\left(N_{t}-N_{s}\right)} \mid \mathcal{F}_{s}^{N} \vee \mathcal{F}_{\infty}^{\lambda}\right)=e^{\left(e^{i u}-1\right) \int_{s}^{t} \lambda_{v} d v} .
$$

This definition implies that

$$
\mathbf{P}\left(N_{t}-N_{s}=k \mid \mathcal{F}_{s}^{N} \vee \mathcal{F}_{\infty}^{\lambda}\right)=\frac{\left(\int_{s}^{t} \lambda_{u} d u\right)^{k}}{k !} e^{-\int_{s}^{t} \lambda_{u} d u} .
$$

Now, we define the rating migration process $C$ by the formula

$$
C_{t}:=\bar{C}_{N_{t}} \text {. }
$$

In this model the Cox process generates impulses in time. Each impulse can cause a rating change, the rating migration process jumps from the current rating $i$ to the rating $j$ with probability $p_{i, j}$ and stays in the current rating $i$ with probability $p_{i, i}$. The intensity of transition from the state $i$ has the form

$$
-\Lambda_{i, i}(t)=\left(1-p_{i, i}\right) \lambda(t) .
$$


The probability of change of rating from the state $i$ to the state $j$, given that the rating changes at time $t$, is equal to

$$
-\frac{\Lambda_{i, j}(t)}{\Lambda_{i, i}(t)}=\frac{p_{i, j} \lambda(t)}{\left(1-p_{i, i}\right) \lambda(t)}=\frac{p_{i, j}}{\left(1-p_{i, i}\right)} .
$$

In this section we make Assumption 3 and the following assumption

Assumption 4. The processes $\left(\bar{C}_{k}\right)_{k \geq 0}$ and $\left(N_{t}\right)_{t \geq 0}$ are conditionally independent given $\mathcal{F}_{\infty}$.

It is easy to see that an appropriate specification of the $P$ matrix ensures that Assumption 3 holds.

In the next theorem we prove that the process $\left(C_{t}\right)_{t \geq 0}$ satisfies properties which are equivalent to Assumptions 1 and 2 from the previous section. Thus, the process $\left(C_{t}\right)_{t \geq 0}$ satisfies Assumption 1, 2, 3, so we can use the results from the previous sections to investigate properties of a model with rating migrations induced by a Cox process.

THEOREM 7. The proces $C$ defined by the formula (22) has the following properties:

i) For every $t \geq 0$ and for every bounded $\mathcal{F}^{C, t}:=\sigma\left\{C_{u}: u \geq t\right\}$ measurable random variable $X$,

$$
\mathbf{E}\left(X \mid \mathcal{F}_{\infty} \vee \mathcal{F}_{t}^{C}\right)=\mathbf{E}\left(X \mid \mathcal{F}_{\infty} \vee \sigma\left(C_{t}\right)\right)
$$

ii) For any $n \in \mathbf{N},\left(i_{1}, \ldots, i_{n}\right) \in \mathcal{K}^{n}$ and all $u_{1} \leq \ldots \leq u_{n} \leq t$ we have

$$
\mathbf{P}\left(C_{u_{1}}=i_{1}, \ldots, C_{u_{n}}=i_{n} \mid \mathcal{F}_{t}\right)=\mathbf{P}\left(C_{u_{1}}=i_{1}, \ldots, C_{u_{n}}=i_{n} \mid \mathcal{F}_{\infty}\right) .
$$

Proof. i) Fix an arbitrary $t \geq 0$, and let $\mathcal{L}$ be a class of sets satisfying

$$
\mathbf{E}\left(\mathbf{1}_{A} \mid \mathcal{F}_{\infty} \vee \mathcal{F}_{t}^{C}\right)=\mathbf{E}\left(\mathbf{1}_{A} \mid \mathcal{F}_{\infty} \vee \sigma\left(C_{t}\right)\right) .
$$

The class $\mathcal{L}$ is a $\lambda$-system. Consider a class $\mathcal{P}_{t}$ of sets of the following form

$$
\begin{array}{r}
\mathcal{P}_{t}:=\left\{A \in \mathcal{F}: A=\left\{C_{u_{1}}=j_{1}, \ldots, C_{u_{n}}=j_{n}\right\} \text { for } \quad \text { some } n \in \mathbf{N},\left(j_{1}, \ldots, j_{n}\right) \in \mathcal{K}^{n},\right. \\
\left.\left(u_{1}, \ldots, u_{n}\right) \in[0, \infty)^{n}, \min \left\{u_{1}, \ldots, u_{n}\right\} \geq t\right\} .
\end{array}
$$

Obviously, $\mathcal{P}_{t}$ is a $\pi$-system. In Lemma 23 in the appendix we prove that equality (23) holds for sets $A$ from the class $\mathcal{P}_{t}$. So $\mathcal{P}_{t} \subseteq \mathcal{L}$ and applying the lemma on $\pi-\lambda$ systems we get that equality (23) holds on sets $A$ from $\sigma\left(\mathcal{P}_{t}\right)=\mathcal{F}^{C, t}:=\sigma\left\{C_{u}: u \geq t\right\}$. Applying the monotone class theorem we obtain that equality (23) holds for an arbitrary bounded $\mathcal{F}^{C, t}$ measurable random variable, which is precisely the assertion i).

ii) We will prove this fact in the appendix in Lemma 22 part b).

Corollary 8. The process $C$ given by $(22)$ is an $\left(\mathbb{F}, \mathbb{F} \vee \mathbb{F}^{C}\right)$ conditional Markov chain.

3.1. Transition probabilities. For an absorbing Markov chain with one absorbing state the canonical form of the transition matrix $P$ has the form

$$
P=\left[\begin{array}{c|c}
Q & R \\
\hline \mathbf{0} & 1
\end{array}\right],
$$


where

$$
\begin{aligned}
Q & =\left[\begin{array}{ccccc}
p_{1,1} & p_{1,2} & p_{1,3} & \ldots & p_{1, K-1} \\
p_{2,1} & p_{2,2} & p_{2,3} & \ldots & p_{2, K-1} \\
p_{3,1} & p_{3,2} & p_{3,3} & \ldots & p_{3, K-1} \\
\vdots & \vdots & \vdots & \ddots & \vdots \\
p_{K-1,1} & p_{K-1,2} & p_{K-1,2} & \ldots & p_{K-1, K-1}
\end{array}\right], \quad R=\left[\begin{array}{c}
p_{1, K} \\
p_{2, K} \\
p_{3, K} \\
\vdots \\
p_{K-1, K}
\end{array}\right], \\
\mathbf{0} & =\left[\begin{array}{llllll}
0, & 0 & 0 & \ldots, & 0
\end{array}\right] .
\end{aligned}
$$

The matrix $P$ is stochastic, so it is uniquely determined by the matrix $Q$ (since $R=$ $(\mathbb{I}-Q) \mathbf{1})$.

From now on, the element $a_{i, j}$ of an arbitrary matrix $A=\left[a_{i, j}\right]_{i, j=1}^{K}$ will be denoted by $[A]_{i, j}$.

THEOREM 9. Let $\left(C_{t}\right)_{t \geq 0}$ be a process given by the formula $(22)$. Then $\tilde{p}_{i, j}(s, t)$ defined by (6) is given by

$$
\tilde{p}_{i, j}(s, t)=\left[e^{(P-I) \int_{s}^{t} \lambda_{u} d u}\right]_{i, j}
$$

Moreover, for $i, j \in \mathcal{K} \backslash K$

$$
\begin{aligned}
\tilde{p}_{i, j}(s, t) & =\left[e^{-(I-Q) \int_{s}^{t} \lambda_{u} d u}\right]_{i, j} \\
& =\sum_{l=1}^{k} \sum_{m=n_{l}^{\prime}+1}^{n_{l}^{\prime}+n_{l}} a_{i, m}\left(\sum_{p=m}^{n_{l}^{\prime}+n_{l}} b_{p, j} e^{-\left(1-d_{l}\right) \int_{s}^{t} \lambda_{u} d u} \frac{\left(\int_{s}^{t} \lambda_{u} d u\right)^{p-m}}{(p-m) !}\right),
\end{aligned}
$$

where $Q$ is the matrix from the canonical form of $P$ (see (24)), and $A=\left[a_{i, j}\right]_{i, j=1}^{K-1}$ is the matrix from the Jordan decomposition of $Q$, i.e. a decomposition of the form $Q=A J A^{-1}$ with a nonsingular matrix $A, J=\oplus_{l=1}^{k} J_{n_{l}}\left(d_{l}\right)$ and $J_{n_{l}}\left(d_{l}\right)$ is a Jordan block of dimension $n_{l}$ associated with eigenvalue $d_{l}$ and $n_{1}^{\prime}:=0, n_{l}^{\prime}:=n_{l-1}^{\prime}+n_{l}$ for $l>1$, and $b_{p, j}:=\left[A^{-1}\right]_{p, j}$.

Proof. Using formula (45) from Lemma 22 and (6), we see after some elementary calculations that

$$
\begin{aligned}
\tilde{p}_{i, j}(s, t) & =\sum_{k=0}^{\infty}\left[P^{k}\right]_{i, j} e^{-\int_{s}^{t} \lambda_{v} d v} \frac{\left(\int_{s}^{t} \lambda_{v} d v\right)^{k}}{k !}=e^{-\int_{s}^{t} \lambda_{v} d v}\left[\sum_{k=0}^{\infty} \frac{\left(P \int_{s}^{t} \lambda_{v} d v\right)^{k}}{k !}\right]_{i, j} \\
& =e^{-\int_{s}^{t} \lambda_{v} d v}\left[e^{P \int_{s}^{t} \lambda_{v} d v}\right]_{i, j}=\left[e^{(P-I) \int_{s}^{t} \lambda_{v} d v}\right]_{i, j} .
\end{aligned}
$$

This ends the proof of formula (25). Now, we deduce formula (26). First, notice that the matrix $Q-I$ has a Jordan decomposition $Q-I=A \tilde{J} A^{-1}$, where $\tilde{J}=(J-I)=$ $\oplus_{l=1}^{k} J_{n_{l}}\left(d_{l}-1\right)$. So, it is well known (see Lemma 24) that

$$
e^{-(I-Q) \int_{s}^{t} \lambda_{u} d u}=A\left(\bigoplus_{l=1}^{k} e^{\left(\int_{s}^{t} \lambda_{u} d u\right) J_{n_{l}}\left(d_{l}-1\right)}\right) A^{-1},
$$

where the matrices in the sum have dimensions $n_{l} \times n_{l}, l=1, \ldots, k$, and terms given by

$$
\left[e^{J_{n_{l}}\left(d_{l}-1\right) \int_{s}^{t} \lambda_{u} d u}\right]_{i, j}= \begin{cases}\frac{\left(\int_{s}^{t} \lambda_{u} d u\right)^{j-i}}{(j-i) !} e^{-\left(1-d_{l}\right) \int_{s}^{t} \lambda_{u} d u}, & \text { if } j-i \geq 0 \\ 0, & \text { if } j-i<0\end{cases}
$$


for $i, j \in \mathcal{K} \backslash K$. Hence, for $i, j \in \mathcal{K} \backslash K$

$$
\left[e^{-(I-Q) \int_{s}^{t} \lambda_{u} d u}\right]_{i, j}=\sum_{l=1}^{k} \sum_{m=n_{l}^{\prime}+1}^{n_{l}^{\prime}+n_{l}} a_{i, m}\left(\sum_{p=m}^{n_{l}^{\prime}+n_{l}} b_{p, j} \frac{\left(\int_{s}^{t} \lambda_{u} d u\right)^{p-m}}{(p-m) !} e^{-\left(1-d_{l}\right) \int_{s}^{t} \lambda_{u} d u}\right),
$$

where $n_{1}^{\prime}:=0, n_{l}^{\prime}:=n_{l-1}^{\prime}+n_{l}$, and the proof is complete.

In some cases formula (26) simplifies considerably:

ExAmPle 10. Assume that the matrix $Q$ is diagonalizable i.e. there exist a nonsingular matrix $A$ and a diagonal matrix $D=\operatorname{diag}\left(d_{1}, \ldots, d_{K-1}\right)$ such that $Q=A D A^{-1}$. Then formula (26) simplifies significantly, namely for $i, j \in \mathcal{K} \backslash K$ we have

$$
\left[e^{-(I-Q) \int_{s}^{t} \lambda_{u} d u}\right]_{i, j}=\sum_{n=1}^{K-1} a_{i, n} b_{n, j} e^{-\left(1-d_{n}\right) \int_{s}^{t} \lambda_{u} d u}
$$

where $b_{n, j}:=\left[A^{-1}\right]_{n, j}$.

3.2. Joint distribution of a default time and a pre-default state of rating. In this subsection we show how to calculate a joint conditional distribution of a default time $\tau$ and a pre-default state of the rating migration process $C$ in terms of the transition matrix $P$ as well as the matrix from the canonical decomposition of $P$.

THEOREM 11. Assume that the process $C$ is given by $(22)$ and $i, j \in \mathcal{K} \backslash K$. Then for any $v>0$ and every $0 \leq t \leq v$, we have

$$
\begin{aligned}
& \mathbf{P}\left(t<\tau \leq v, C_{\tau-}=j \mid \mathcal{F}_{\infty} \vee \sigma\left(C_{t}\right)\right) \mathbf{1}_{\left\{C_{t}=i\right\}} \\
& =\mathbf{1}_{\left\{C_{t}=i\right\}} e^{-\int_{t}^{v} \lambda_{u} d u} p_{j, K} \sum_{l=1}^{\infty}\left(\sum_{m=1}^{l}\left[P^{m-1}\right]_{i, j}\right) \frac{\left(\int_{t}^{v} \lambda_{u} d u\right)^{l}}{l !} .
\end{aligned}
$$

Proof. By Lemma 3

$$
\mathbf{P}\left(t<\tau \leq v, C_{\tau-}=j \mid \mathcal{F}_{\infty} \vee \sigma\left(C_{t}\right)\right) \mathbf{1}_{\left\{C_{t}=i\right\}}=\frac{\mathbf{P}\left(t<\tau \leq v, C_{\tau-}=j, C_{t}=i \mid \mathcal{F}_{\infty}\right)}{\mathbf{P}\left(C_{t}=i \mid \mathcal{F}_{\infty}\right)} \mathbf{1}_{\left\{C_{t}=i\right\}}
$$

Let

$$
A:=\left\{C_{\tau-}=j, C_{t}=i, t<\tau \leq v\right\} .
$$

Our task is to derive $\mathbf{P}\left(A \mid \mathcal{F}_{\infty}\right)$ in terms of the matrix $P$ and the intensity process $\left(\lambda_{t}\right)_{t \geq 0}$. Since the process $C$ is defined by (22) we have

$$
A=\bigcup_{l=0}^{\infty}\left\{C_{\tau-}=j, C_{t}=i, t<\tau \leq v, N_{v}-N_{t}=l\right\}
$$

and

$$
\left\{C_{t}=i\right\}=\bigcup_{k=0}^{\infty}\left\{\bar{C}_{k}=i, N_{t}=k\right\}
$$


Therefore, we can represent the event $A$ in the following way

$$
\begin{aligned}
A & =\bigcup_{k=0}^{\infty} \bigcup_{l=1}^{\infty}\left(\left\{N_{t}=k\right\} \cap\left\{N_{v}-N_{t}=l\right\} \cap \bigcup_{m=1}^{l}\left\{C_{\gamma_{m+k}}=K, C_{\gamma_{m-1+k}}=j, C_{\gamma_{k}}=i\right\}\right) \\
& =\bigcup_{k=0}^{\infty} \bigcup_{l=1}^{\infty}\left(\left\{N_{t}=k, N_{v}-N_{t}=l\right\} \cap \bigcup_{m=1}^{l}\left\{\bar{C}_{m+k}=K, \bar{C}_{m-1+k}=j, \bar{C}_{k}=i\right\}\right),
\end{aligned}
$$

where by $\left(\gamma_{n}\right)_{n \geq 0}$ we denote the subsequent jumps of the Cox process $\left(N_{t}\right)_{t \geq 0}$. Using this form of the event $A$ we conclude that

$$
\begin{aligned}
\mathbf{P} & \left(A \mid \mathcal{F}_{\infty}\right) \\
= & \mathbf{P}\left(\bigcup_{k=0}^{\infty} \bigcup_{l=1}^{\infty}\left(\left\{N_{t}=k, N_{v}-N_{t}=l\right\} \cap \bigcup_{m=1}^{l}\left\{\bar{C}_{m+k}=K, \bar{C}_{m-1+k}=j, \bar{C}_{k}=i\right\}\right) \mid \mathcal{F}_{\infty}\right) \\
= & \sum_{k=0}^{\infty} \sum_{l=1}^{\infty} \mathbf{P}\left(\left\{N_{t}=k, N_{v}-N_{t}=l\right\} \cap \bigcup_{m=1}^{l}\left\{\bar{C}_{m+k}=K, \bar{C}_{m-1+k}=j, \bar{C}_{k}=i\right\} \mid \mathcal{F}_{\infty}\right) \\
= & \sum_{k=0}^{\infty} \sum_{l=1}^{\infty} \mathbf{P}\left(N_{t}=k, N_{v}-N_{t}=l \mid \mathcal{F}_{\infty}\right) \mathbf{P}\left(\bigcup_{m=1}^{l}\left\{\bar{C}_{m+k}=K, \bar{C}_{m-1+k}=j, \bar{C}_{k}=i\right\} \mid \mathcal{F}_{\infty}\right) \\
= & \sum_{k=0}^{\infty} \sum_{l=1}^{\infty} \mathbf{P}\left(\bigcup_{m=1}^{l}\left\{\bar{C}_{m+k}=K, \bar{C}_{m-1+k}=j, \bar{C}_{k}=i\right\} \mid \mathcal{F}_{\infty}\right) e^{-\int_{t}^{v} \lambda_{u} d u} \frac{\left(\int_{t}^{v} \lambda_{u} d u\right)^{l}}{l !} \\
& \times e^{-\int_{0}^{t} \lambda_{u} d u} \frac{\left(\int_{0}^{t} \lambda_{u} d u\right)^{k}}{k !},
\end{aligned}
$$

where the third equality follows from Assumption 4. Independence of the Markov chain $\left(\bar{C}_{k}\right)_{k \geq 0}$ and the $\sigma$-field $\mathcal{F}_{\infty}$ implies that

$$
\begin{aligned}
& \mathbf{P}\left(\bigcup_{m=1}^{l}\left\{\bar{C}_{m+k}=K, \bar{C}_{m-1+k}=j, \bar{C}_{k}=i\right\} \mid \mathcal{F}_{\infty}\right) \\
& =\mathbf{P}\left(\bigcup_{m=1}^{l}\left\{\bar{C}_{m+k}=K, \bar{C}_{m-1+k}=j, \bar{C}_{k}=i\right\}\right) \\
& =\sum_{m=1}^{l} \mathbf{P}\left(\bar{C}_{m+k}=K \mid \bar{C}_{m-1+k}=j\right) \mathbf{P}\left(\bar{C}_{m-1+k}=j \mid \bar{C}_{k}=i\right) \mathbf{P}\left(\bar{C}_{k}=i \mid \bar{C}_{0}=i_{0}\right) \\
& =p_{j, K}\left[P^{k}\right]_{i_{0}, i} \sum_{m=1}^{l}\left[P^{m-1}\right]_{i, j} .
\end{aligned}
$$

Substituting this expression into the formula (30) we obtain

$$
\begin{aligned}
& \mathbf{P}\left(A \mid \mathcal{F}_{\infty}\right) \\
& =\sum_{k=0}^{\infty} \sum_{l=1}^{\infty}\left(p_{j, K}\left[P^{k}\right]_{i_{0}, i} \sum_{m=1}^{l}\left[P^{m-1}\right]_{i, j}\right) e^{-\int_{t}^{v} \lambda_{u} d u} \frac{\left(\int_{t}^{v} \lambda_{u} d u\right)^{l}}{l !} e^{-\int_{0}^{t} \lambda_{u} d u} \frac{\left(\int_{0}^{t} \lambda_{u} d u\right)^{k}}{k !}
\end{aligned}
$$




$$
\begin{aligned}
= & \left(\sum_{k=0}^{\infty}\left[P^{k}\right]_{i_{0}, i} e^{-\int_{0}^{t} \lambda_{u} d u} \frac{\left(\int_{0}^{t} \lambda_{u} d u\right)^{k}}{k !}\right) \\
& \times\left(\sum_{l=1}^{\infty}\left(p_{j, K} \sum_{m=1}^{l}\left[P^{m-1}\right]_{i, j}\right) e^{-\int_{t}^{v} \lambda_{u} d u} \frac{\left(\int_{t}^{v} \lambda_{u} d u\right)^{l}}{l !}\right) \\
= & \mathbf{P}\left(C_{t}=i \mid \mathcal{F}_{\infty}\right)\left(\sum_{l=1}^{\infty}\left(p_{j, K} \sum_{m=1}^{l}\left[P^{m-1}\right]_{i, j}\right) e^{-\int_{t}^{v} \lambda_{u} d u} \frac{\left(\int_{t}^{v} \lambda_{u} d u\right)^{l}}{l !}\right),
\end{aligned}
$$

where the last equality follows from Lemma 22 (formula (45)). Putting the derived formula for $\mathbf{P}\left(A \mid \mathcal{F}_{\infty}\right)$ into (29) we obtain the desired result.

Since

$$
\begin{aligned}
\mathbf{1}_{\left\{C_{t}=i\right\}} G_{t, i, j}(v) & =\mathbf{1}_{\left\{C_{t}=i\right\}} \frac{\mathbf{P}\left(\tau \leq v, C_{t}=i, C_{\tau-}=j \mid \mathcal{F}_{\infty}\right)}{\mathbf{P}\left(C_{t}=i \mid \mathcal{F}_{\infty}\right)} \\
& =\mathbf{P}\left(t<\tau \leq v, C_{\tau-}=j \mid \mathcal{F}_{\infty} \vee \sigma\left(C_{t}\right)\right) \mathbf{1}_{\left\{C_{t}=i\right\}},
\end{aligned}
$$

we see that the previous theorem is useful in deriving the probabilities $G_{t, i, j}(v)$. In the next theorem we derive a formula for $G_{t, i, j}(v)$ in terms of some matrix exponent given in terms of the matrix $Q$.

THEOREM 12. Let $Q$ be the matrix from the canonical decomposition of $P$ and $i, j \in \mathcal{K} \backslash K$. Then for any $v>0$ and for every $0 \leq t \leq v$ we have

$$
\begin{aligned}
& \mathbf{P}\left(t<\tau \leq v, C_{\tau-}=j \mid \mathcal{F}_{\infty} \vee \sigma\left(C_{t}\right)\right) \mathbf{1}_{\left\{C_{t}=i\right\}} \\
& \quad=\mathbf{1}_{\left\{C_{t}=i\right\}} p_{j, K}\left[(\mathbb{I}-Q)^{-1}\left(\mathbb{I}-e^{-(\mathbb{I}-Q) \int_{t}^{v} \lambda_{u} d u}\right)\right]_{i, j} .
\end{aligned}
$$

Proof. Set

$$
I:=e^{-\int_{t}^{v} \lambda_{u} d u} p_{j, K} \sum_{l=1}^{\infty}\left(\sum_{m=1}^{l}\left[P^{m-1}\right]_{i, j}\right) \frac{\left(\int_{t}^{v} \lambda_{u} d u\right)^{l}}{l !} .
$$

Taking into account (28), it is enough to prove that

$$
I=p_{j, K}\left[(\mathbb{I}-Q)^{-1}\left(\mathbb{I}-e^{-(\mathbb{I}-Q) \int_{t}^{v} \lambda_{u} d u}\right)\right]_{i, j} .
$$

First of all, notice that for any $k \geq 0$ we have

$$
P^{k}=\left[\begin{array}{c|c}
Q^{k} & \left(\mathbb{I}-Q^{k}\right) \mathbf{1} \\
\hline \mathbf{0} & 1
\end{array}\right] .
$$

Hence, for $i, j \in \mathcal{K} \backslash K$, we obtain

$$
I=p_{j, K}\left[\sum_{l=1}^{\infty}\left(\sum_{m=1}^{l} Q^{m-1}\right) \frac{\left(\int_{t}^{v} \lambda_{u} d u\right)^{l}}{l !} e^{-\int_{t}^{v} \lambda_{u} d u}\right]_{i, j} .
$$

For absorbing Markov chains with one absorbing state and with remaining state transient, $(\mathbb{I}-Q)^{-1}$ exists (see e.g. Grinstead and Snell [10]) and as a consequence we obtain

$$
\sum_{m=1}^{l} Q^{m-1}=(\mathbb{I}-Q)^{-1}\left(\mathbb{I}-Q^{l}\right) .
$$


Therefore

$$
\begin{aligned}
I & =p_{j, K}\left[\sum_{l=1}^{\infty}(\mathbb{I}-Q)^{-1}\left(\mathbb{I}-Q^{l}\right) \frac{\left(\int_{t}^{v} \lambda_{u} d u\right)^{l}}{l !} e^{-\int_{t}^{v} \lambda_{u} d u}\right]_{i, j} \\
& =p_{j, K} e^{-\int_{t}^{v} \lambda_{u} d u}\left[(\mathbb{I}-Q)^{-1}\left(\sum_{l=1}^{\infty} \mathbb{I} \frac{\left(\int_{t}^{v} \lambda_{u} d u\right)^{l}}{l !}-\sum_{l=1}^{\infty} \frac{\left(Q \int_{t}^{v} \lambda_{u} d u\right)^{l}}{l !}\right)\right]_{i, j} \\
& =p_{j, K} e^{-\int_{t}^{v} \lambda_{u} d u}\left[(\mathbb{I}-Q)^{-1}\left(\mathbb{I} e^{\int_{t}^{v} \lambda_{u} d u}-\mathbb{I}-\left(e^{Q \int_{t}^{v} \lambda_{u} d u}-\mathbb{I}\right)\right)\right]_{i, j} \\
& =p_{j, K} e^{-\int_{t}^{v} \lambda_{u} d u}\left[(\mathbb{I}-Q)^{-1}\left(\mathbb{I} e^{\int_{t}^{v} \lambda_{u} d u}-e^{Q \int_{t}^{v} \lambda_{u} d u}\right)\right]_{i, j} \\
& =p_{j, K}\left[(\mathbb{I}-Q)^{-1}\left(\mathbb{I}-e^{-(\mathbb{I}-Q) \int_{t}^{v} \lambda_{u} d u}\right)\right]_{i, j},
\end{aligned}
$$

which completes the proof.

Using Theorem 12, the definition of $G$ and (31) we can find a compact form of the differential $d G_{t, i, j}(v)$.

COROLlary 13. The differential $d G_{t, i, j}(v)$ has the following form

$$
d G_{t, i, j}(v)=\left[e^{-(\mathbb{I}-Q) \int_{t}^{v} \lambda_{u} d u}\right]_{i, j} p_{j, K} \lambda_{v} d v .
$$

\section{Pricing of bonds and credit default swaps}

4.1. Pricing of defaultable bonds. We consider the defaultable bond with fractional recovery of par value, maturing at time $T$, which is subject to a rating migration according to the process $C$ defined by the formula (22). The default time is defined as before, i.e. as the first moment of reaching the absorbing state $K$ by $C$, i.e.,

$$
\tau:=\inf \left\{t>0: C_{t}=K\right\} .
$$

Such bond pays 1 unit of cash at maturity time $T$ provided that a credit event occurred after $T$ (i.e. on the set $\{\tau>T\}$ ). While on the set $\{\tau \leq T\}$ the bond pays at the default moment $\tau$ a recovery payment $\delta_{C_{\tau-}} \in[0,1)$ which is some fraction of its nominal value $\left(\delta_{j}\right.$ is a fixed number for each $j \in \mathcal{K} \backslash K$ ). It is important to notice that a recovery payment depends on $C_{\tau-}$, i.e. on the rating of bond before the default time. Under the assumption of absence of arbitrage opportunities, the price (the ex-dividend price) of such defaultable bond is expressed by the following conditional expectation

$$
D(t, T)=B_{t} \mathbf{E}\left(\frac{1}{B_{T}} \mathbf{1}_{\{\tau>T\}}+\frac{\delta_{C_{\tau-}}}{B_{\tau}} \mathbf{1}_{\{t<\tau \leq T\}} \mid \mathcal{G}_{t}\right),
$$

where $\left(B_{t}\right)_{t \geq 0}$ is the value of the saving account. Of course, the short rate process is $\mathbb{F}$-adapted and will be denoted by $\left(r_{t}\right)_{t \geq 0}$, so $B_{t}=\exp \left(\int_{0}^{t} r_{s} d s\right)$. In this section, we assume that $\mathbb{G}=\mathbb{F} \vee \mathbb{F}^{C}$. Now, applying formulae for conditional expectations from section 2 we can calculate the price of bonds under consideration.

THEOREM 14. Assume that $C$ is given by (22) with intensity process $\left(\lambda_{u}\right)_{u \geq 0}$ and transition matrix $P$. For $t<T$, the price of a defaultable bond with fractional recovery of par value is equal to 


$$
\begin{aligned}
D(t, T) \mathbf{1}_{\{\tau>t\}}=\sum_{i=1}^{K-1} \mathbf{1}_{\left\{C_{t}=i\right\}} & {\left[\sum _ { j = 1 } ^ { K - 1 } \left(\mathbf{E}\left(e^{-\int_{t}^{T} r_{u} d u}\left[e^{-(\mathbb{I}-Q) \int_{t}^{T} \lambda_{u} d u}\right]_{i, j} \mid \mathcal{F}_{t}\right)\right.\right.} \\
& \left.\left.+\delta_{j} \int_{t}^{T} \mathbf{E}\left(e^{-\int_{t}^{u} r_{v} d v}\left[e^{-(\mathbb{I}-Q) \int_{t}^{u} \lambda_{v} d v}\right]_{i, j} p_{j, K} \lambda_{u} \mid \mathcal{F}_{t}\right) d u\right)\right] .
\end{aligned}
$$

Proof. For $i \in \mathcal{K} \backslash K$ we have

$$
\begin{aligned}
D(t, T) \mathbf{1}_{\left\{C_{t}=i\right\}} & =\mathbf{1}_{\left\{C_{t}=i\right\}} B_{t} \mathbf{E}\left(\frac{1}{B_{T}} \mathbf{1}_{\{\tau>T\}}+\frac{\delta_{C_{\tau-}}}{B_{\tau}} \mathbf{1}_{\{t<\tau \leq T\}} \mid \mathcal{G}_{t}\right) \\
& =\mathbf{1}_{\left\{C_{t}=i\right\}} B_{t} \mathbf{E}\left(\frac{1}{B_{T}} \mathbf{1}_{\left\{C_{T} \neq K\right\}}+\frac{\delta_{C_{\tau-}}}{B_{\tau}} \mathbf{1}_{\{t<\tau \leq T\}} \mid \mathcal{G}_{t}\right) \\
& =\mathbf{1}_{\left\{C_{t}=i\right\}} \sum_{j=1}^{K-1} B_{t} \mathbf{E}\left(\frac{1}{B_{T}} \mathbf{1}_{\left\{C_{T}=j\right\}}+\frac{\delta_{j}}{B_{\tau}} \mathbf{1}_{\left\{t<\tau \leq T, C_{\tau-}=j, C_{t}=i\right\}} \mid \mathcal{G}_{t}\right) .
\end{aligned}
$$

The process $\left(B_{t}\right)_{t \geq 0}$ is $\mathbb{F}$-predictable, by assumption, and therefore application of Corollary 5 and Theorem 6 enables us to write

$$
D(t, T) \mathbf{1}_{\left\{C_{t}=i\right\}}=\mathbf{1}_{\left\{C_{t}=i\right\}} \sum_{j=1}^{K-1} \mathbf{E}\left(\frac{B_{t}}{B_{T}} \tilde{p}_{i, j}(t, T)+\delta_{j} \int_{t}^{T} \frac{B_{t}}{B_{u}} d G_{t, i, j}(u) \mid \mathcal{F}_{t}\right)=I .
$$

Using formulae (26) and (33) and the conditional stochastic Fubini theorem (see Applebaum [2]) we obtain

$$
\begin{aligned}
I=\mathbf{1}_{\left\{C_{t}=i\right\}} \sum_{j=1}^{K-1}\left(\mathbf{E}\left(e^{-\int_{t}^{T} r_{u} d u}\left[e^{-(\mathbb{I}-Q) \int_{t}^{T} \lambda_{u} d u}\right]_{i, j} \mid \mathcal{F}_{t}\right)\right. \\
\left.+\delta_{j} \int_{t}^{T} \mathbf{E}\left(e^{-\int_{t}^{u} r_{v} d v}\left[e^{-(\mathbb{I}-Q) \int_{t}^{u} \lambda_{v} d v}\right]_{i, j} p_{j, K} \lambda_{u} \mid \mathcal{F}_{t}\right) d u\right),
\end{aligned}
$$

which establishes the formula.

Using Theorem 14 and Theorem 9 (equality (26)) we can express the price of a defaultable bond in explicit form.

COROLlaRY 15. The price of a defaultable bond with fractional recovery of par value is equal to

$$
\begin{array}{r}
D(t, T) \mathbf{1}_{\left\{C_{t}=i\right\}}=\mathbf{1}_{\left\{C_{t}=i\right\}} \sum_{j=1}^{K-1} \sum_{l=1}^{k} \sum_{m=n_{l}^{\prime}+1}^{n_{l}^{\prime}+n_{l}} a_{i, m} \sum_{p=m}^{n_{l}^{\prime}+n_{l}} b_{p, j}\left(H\left(t, T, p-m, 1-d_{l}\right)\right. \\
\left.+\delta_{j} p_{j, K} \int_{t}^{T} B\left(t, u, p-m, 1-d_{l}\right) d u\right)
\end{array}
$$

where:

$$
\begin{aligned}
B(t, u, k, w) & :=\mathbf{E}\left(\frac{\left(\int_{t}^{u} \lambda_{v} d v\right)^{k}}{k !} e^{-\int_{t}^{u}\left[r_{v}+w \lambda_{v}\right] d v} \lambda_{u} \mid \mathcal{F}_{t}\right), \\
H(t, u, k, w) & :=\mathbf{E}\left(\frac{\left(\int_{t}^{u} \lambda_{v} d v\right)^{k}}{k !} e^{-\int_{t}^{u}\left[r_{v}+w \lambda_{v}\right] d v} \mid \mathcal{F}_{t}\right) .
\end{aligned}
$$


Using (27) we obtain

Corollary 16. Assume that the matrix $Q$ is diagonalizable. Then

$$
\begin{aligned}
& D(t, T) \mathbf{1}_{\left\{C_{t}=i\right\}}= \\
& \quad \mathbf{1}_{\left\{C_{t}=i\right\}} \sum_{j=1}^{K-1} \sum_{n=1}^{K-1} a_{i, n} b_{n, j}\left(H\left(t, T, 0,1-d_{n}\right)+\delta_{j} p_{j, K} \int_{t}^{T} B\left(t, u, 0,1-d_{n}\right) d u\right) .
\end{aligned}
$$

4.2. Pricing of Credit Default Swaps. In this subsection, using the previous results we describe a method of finding prices of credit derivatives connected with bonds considered before. We do it considering a Credit Default Swap. The Credit Default Swap (CDS) is an agreement between two counterparties: a protection buyer and a protection seller. This agreement has two legs:

Premium Leg: The protection buyer agrees to pay a fixed amount $\kappa$ (CDS spread) on fixed dates in time $\mathcal{T}=\left\{T_{1}<T_{2}<\ldots<T_{n}\right\}$. He pays $\kappa$ at time $T_{k}$ provided that default does not occur before or at $T_{k}$. Then, for $t<T_{1}$ the value of the premium leg is equal to

$$
V_{P}(t)=B_{t} \mathbf{E}\left(\sum_{k=1}^{n} \frac{\kappa}{B_{T_{k}}} \mathbf{1}_{\left\{\tau>T_{k}\right\}} \mid \mathcal{G}_{t}\right)
$$

Default Leg: The protection seller agrees to cover all losses connected with this bond provided that loss occurs before the protection horizon $T_{n}$. For $t<T_{n}$, the value of this default leg is equal to

$$
V_{D}(t)=B_{t} \mathbf{E}\left(\frac{1-\delta_{C_{\tau-}}}{B_{\tau}} \mathbf{1}_{\left\{t<\tau \leq T_{n}\right\}} \mid \mathcal{G}_{t}\right)
$$

If we know the value of the spread, i.e. $\kappa$, then the CDS value at time $t$ is the difference between the premium leg and default leg

$$
\operatorname{CDS}(t, \mathcal{T}, \kappa)=V_{P}(t)-V_{D}(t)=B_{t} \mathbf{E}\left(\sum_{k=1}^{n} \frac{\kappa}{B_{T_{k}}} \mathbf{1}_{\left\{\tau>T_{k}\right\}}-\frac{\left(1-\delta_{C_{\tau-}}\right)}{B_{\tau}} \mathbf{1}_{\left\{t<\tau \leq T_{n}\right\}} \mid \mathcal{G}_{t}\right) .
$$

The market CDS spread $\kappa$ (sometimes also called the "fair spread") is determined at the contract inception date $t<T_{1}$ in such a way that the value of the contract is 0 , i.e.

$$
\operatorname{CDS}(t, \mathcal{T}, \kappa)=0 \text {. }
$$

Pricing of CDS is mainly the issue of calculation of the fair spread $\kappa(t, \mathcal{T})$. In order to do this we have to be able to calculate the value of both legs, since the fair CDS spread is given as

$$
\kappa(t, \mathcal{T}) \mathbf{1}_{\{\tau>t\}}=\mathbf{1}_{\{\tau>t\}} \frac{\mathbf{E}\left(\frac{B_{t}}{B_{\tau}}\left(1-\delta_{C_{\tau-}}\right) \mathbf{1}_{\left\{t<\tau \leq T_{n}\right\}} \mid \mathcal{G}_{t}\right)}{\mathbf{E}\left(\sum_{k=1}^{n} \frac{B_{t}}{B_{T_{k}}} \mathbf{1}_{\left\{\tau>T_{k}\right\}} \mid \mathcal{G}_{t}\right)} .
$$

The next theorem provides formulae for the value of both legs expressed through an exponential involving the matrix $(Q-I)$ and the intensity process $\left(\lambda_{t}\right)_{t \geq 0}$.

Theorem 17. Assume that $C$ is given by (22) with intensity process $\left(\lambda_{u}\right)_{u \geq 0}$ and transition matrix $P$. Let $t<T_{1}$. The value of the default leg is equal to

$$
V_{D}(t)=\sum_{i=1}^{K-1} \mathbf{1}_{\left\{C_{t}=i\right\}}\left(\sum_{j=1}^{K-1}\left(1-\delta_{j}\right) \int_{t}^{T_{n}} \mathbf{E}\left(e^{-\int_{t}^{u} r_{v} d v}\left[e^{-(\mathbb{I}-Q) \int_{t}^{u} \lambda_{v} d v}\right]_{i, j} p_{j, K} \lambda_{u} \mid \mathcal{F}_{t}\right) d u\right) .
$$


The value of the premium leg is given by

$$
V_{P}(t)=\sum_{i=1}^{K-1} \mathbf{1}_{\left\{C_{t}=i\right\}}\left(\sum_{k=1}^{n} \sum_{j=1}^{K-1} \mathbf{E}\left(e^{-\int_{t}^{T_{k}} r_{u} d u}\left[e^{-(\mathbb{I}-Q) \int_{t}^{T_{k}} \lambda_{u} d u}\right]_{i, j} \mid \mathcal{F}_{t}\right)\right) .
$$

Proof. Let us first consider the default leg. For each $i \in \mathcal{K} \backslash K$ we have

$$
\begin{aligned}
V_{D}(t) \mathbf{1}_{\left\{C_{t}=i\right\}} & =B_{t} \mathbf{E}\left(\frac{1-\delta_{C_{\tau-}}}{B_{\tau}} \mathbf{1}_{\left\{t<\tau \leq T_{n}\right\}} \mid \mathcal{G}_{t}\right) \mathbf{1}_{\left\{C_{t}=i\right\}} \\
& =\mathbf{1}_{\left\{C_{t}=i\right\}} B_{t} \sum_{j=1}^{K-1} \mathbf{E}\left(\frac{1-\delta_{j}}{B_{\tau}} \mathbf{1}_{\left\{t<\tau \leq T_{n}, C_{\tau}-=j\right\}} \mid \mathcal{G}_{t}\right) \\
& =\mathbf{1}_{\left\{C_{t}=i\right\}}\left(\sum_{j=1}^{K-1}\left(1-\delta_{j}\right) B_{t} \mathbf{E}\left(\int_{t}^{T_{n}} \frac{1}{B_{u}} d G_{t, i, j}(u) \mid \mathcal{F}_{t}\right)\right) \\
& =\mathbf{1}_{\left\{C_{t}=i\right\}}\left(\sum_{j=1}^{K-1}\left(1-\delta_{j}\right) \mathbf{E}\left(\int_{t}^{T_{n}} e^{-\int_{t}^{u} r_{v} d v}\left[e^{-(\mathbb{I}-Q) \int_{t}^{u} \lambda_{v} d v}\right]_{i, j} p_{j, K} \lambda_{u} d u \mid \mathcal{F}_{t}\right)\right) \\
& =\mathbf{1}_{\left\{C_{t}=i\right\}}\left(\sum_{j=1}^{K-1}\left(1-\delta_{j}\right) \int_{t}^{T_{n}} \mathbf{E}\left(e^{-\int_{t}^{u} r_{v} d v}\left[e^{-(\mathbb{I}-Q) \int_{t}^{u} \lambda_{v} d v}\right]_{i, j} p_{j, K} \lambda_{u} \mid \mathcal{F}_{t}\right) d u\right),
\end{aligned}
$$

where the third equality follows by application of Theorem 6 (the process $\left(B_{t}\right)_{t \geq 0}$ is $\mathbb{F}$-predictable) and the fourth follows from (33).

To obtain the value of the premium leg, let us note that for $i \in \mathcal{K} \backslash K$

$$
\begin{aligned}
& V_{P}(t) \mathbf{1}_{\left\{C_{t}=i\right\}}=\kappa B_{t} \mathbf{E}\left(\sum_{k=1}^{n} \frac{1}{B_{T_{k}}} \mathbf{1}_{\left\{\tau>T_{k}\right\}} \mid \mathcal{G}_{t}\right) \mathbf{1}_{\left\{C_{t}=i\right\}} \\
& =\kappa \sum_{k=1}^{n} \mathbf{E}\left(\frac{B_{t}}{B_{T_{k}}} \mathbf{1}_{\left\{C_{T_{k}} \neq K\right\}} \mid \mathcal{G}_{t}\right) \mathbf{1}_{\left\{C_{t}=i\right\}}=\kappa \sum_{k=1}^{n} \mathbf{E}\left(\frac{B_{t}}{B_{T_{k}}} \sum_{j=1}^{K-1} \tilde{p}_{i, j}\left(t, T_{k}\right) \mid \mathcal{F}_{t}\right) \mathbf{1}_{\left\{C_{t}=i\right\}} \\
& =\kappa \sum_{k=1}^{n} \sum_{j=1}^{K-1} \mathbf{E}\left(e^{-\int_{t}^{T_{k}} r_{u} d u}\left[e^{-(\mathbb{I}-Q) \int_{t}^{T_{k}} \lambda_{u} d u}\right]_{i, j} \mid \mathcal{F}_{t}\right) \mathbf{1}_{\left\{C_{t}=i\right\}},
\end{aligned}
$$

where in the third equality we have used (11) and in the last (25).

Corollary 18. Assume that $C$ is given by expression (22) with intensity process $\left(\lambda_{u}\right)_{u \geq 0}$ and transition matrix $P$. Then the value of the fair CDS spread at time $t<T_{1}$ is given by the formula

$\kappa(t, \mathcal{T}) \mathbf{1}_{\{\tau>t\}}$

$$
=\sum_{i=1}^{K-1} \mathbf{1}_{\left\{C_{t}=i\right\}} \frac{\sum_{j=1}^{K-1}\left(1-\delta_{j}\right) \int_{t}^{T_{n}} \mathbf{E}\left(e^{-\int_{t}^{u} r_{v} d v}\left[e^{-(\mathbb{I}-Q) \int_{t}^{u} \lambda_{v} d v}\right]_{i, j} p_{j, K} \lambda_{u} \mid \mathcal{F}_{t}\right) d u}{\sum_{k=1}^{n} \sum_{j=1}^{K-1} \mathbf{E}\left(e^{-\int_{t}^{T_{k}} r_{u} d u}\left[e^{-(\mathbb{I}-Q) \int_{t}^{T_{k}} \lambda_{u} d u}\right]_{i, j} \mid \mathcal{F}_{t}\right)} .
$$


Using Jordan's decomposition of the matrix $Q$ we obtain by (26) more explicit formulae:

COROLlary 19. The value of the fair CDS spread at date $t<T_{1}$ is given by the formula: $\kappa(t, \mathcal{T}) \mathbf{1}_{\{\tau>t\}}=$

$$
\sum_{i=1}^{K-1} \mathbf{1}_{\left\{C_{t}=i\right\}} \frac{\sum_{j=1}^{K-1} \sum_{l=1}^{k} \sum_{m=n_{l}^{\prime}+1}^{n_{l}^{\prime}+n_{l}} \sum_{p=m}^{n_{l}^{\prime}+n_{l}} a_{i, m} b_{p, j}\left(1-\delta_{j}\right) p_{j, K} \int_{t}^{T_{n}} B\left(t, u, p-m, 1-d_{l}\right) d u}{\sum_{k=1}^{n} \sum_{j=1}^{K-1} \sum_{l=1}^{k} \sum_{m=n_{l}^{\prime}+1}^{n_{l}^{\prime}+n_{l}} \sum_{p=m}^{n_{l}^{\prime}+n_{l}} a_{i, m} b_{p, j} H\left(t, T_{k}, p-m, 1-d_{l}\right)},
$$

where $B$ and $C$ are defined by (34) and (35) respectively.

Corollary 20. Suppose that the matrix $Q$ is diagonalizable. Then

$$
\kappa(t, \mathcal{T}) \mathbf{1}_{\{\tau>t\}}=\sum_{i=1}^{K-1} \mathbf{1}_{\left\{C_{t}=i\right\}} \frac{\sum_{j=1}^{K-1} \sum_{l=1}^{K-1} a_{i, l} b_{l, j}\left(1-\delta_{j}\right) p_{j, K} \int_{t}^{T_{n}} B\left(t, u, 0,1-d_{l}\right) d u}{\sum_{k=1}^{n} \sum_{j=1}^{K-1} \sum_{l=1}^{K-1} a_{i, l} b_{l, j} H\left(t, T_{k}, 0,1-d_{l}\right)},
$$

where $B$ and $C$ are defined by (34) and (35) respectively.

Proof. This is a simple consequence of the previous results and (27).

4.3. Example. In this subsection we provide an example of a specification of the shortrate process $\left(r_{t}\right)_{t \geq 0}$ and intensity process $\left(\lambda_{t}\right)_{t \geq 0}$, under which the conditional transforms defined by (34) and (35), i.e. the functions $B$ and $C$, have an explicit form. Therefore, prices of defaultable bonds with fractional recovery of par value and prices of CDS have closed forms in the model with such short-rate and intensity processes.

Assume that the processes $r$ and $\lambda$ are the solution of a general Ornstein-Uhlenbeck equation with Lévy noise, i.e.:

$$
\begin{gathered}
d r(t)=\theta_{r}\left(\kappa_{r}-r(t-)\right) d t+\left\langle\sigma_{r}, d Z(t)\right\rangle, \\
d \lambda(t)=\theta_{\lambda}\left(\kappa_{\lambda}-\lambda(t-)\right) d t+\left\langle\sigma_{\lambda}, d Z(t)\right\rangle,
\end{gathered}
$$

where the coefficients $\theta_{r}, \kappa_{r}, \theta_{\lambda}, \kappa_{\lambda}$ are positive constants, $\sigma_{r}, \sigma_{\lambda} \in \mathbb{R}_{+}^{2}$ and $Z$ is a twodimensional pure jump Lévy process with values in $\mathbb{R}_{+}^{2}$ and the Lévy measure denoted by $\nu$.

Now, we quote the theorem which describes explicit forms of the transforms defined by (34) and (35) (for the proof see Niewęgłowski [18]). We start from

Definition 2. The point $(w, v)$ is called admissible for parameters $\left(\theta_{r}, \theta_{\lambda}, \sigma_{r}, \sigma_{\lambda}, \nu\right)$ if for all $t \in[0, u]$

$$
-\frac{\sigma_{r}}{\theta_{r}}\left(e^{-\theta_{r}(u-t)}-1\right)+\sigma_{\lambda}\left(v e^{-\theta_{\lambda}(u-t)}-\frac{w}{\theta_{\lambda}}\left(e^{-\theta_{\lambda}(u-t)}-1\right)\right) \in \operatorname{Int} B,
$$

where

$$
B:=\left\{x: \int_{|y|>1} e^{-\langle x, y\rangle} \nu(d y)<\infty\right\} .
$$

The notion of admissibility gives a sufficient condition for existence of integrals in the following theorem. 
THEOREM 21 ([18]). Let a point $(w, v)$ be admissible for parameters $\left(\theta_{r}, \theta_{\lambda}, \sigma_{r}, \sigma_{\lambda}, \nu\right)$. Then for $t \in[0, u]$ we have

$$
\mathbf{E}\left(e^{-\int_{t}^{u}[r(s)+w \lambda(s)] d s} e^{-v \lambda(u)} \mid \mathcal{F}_{t}\right)=e^{-B_{r}(t, u) r(t)-B_{\lambda}(t, u, w, v) \lambda(t)+A(t, u, w, v)},
$$

where $B_{r}(t, u), B_{\lambda}(t, u, w, v), A(t, u, w, v)$ are real valued functions in $t$ for fixed $u, w, v$ and they are solution on the interval $[0, u]$ to the following system of ODE:

$$
\begin{aligned}
& 0=-\frac{\partial B_{r}(t)}{\partial t}+B_{r}(t) \theta_{r}-1 \\
& 0=-\frac{\partial B_{\lambda}(t)}{\partial t}+B_{\lambda}(t) \theta_{\lambda}-w \\
& 0=\frac{\partial A(t)}{\partial t}-\theta_{r} \kappa_{r} B_{r}(t)-\theta_{\lambda} \kappa_{\lambda} B_{\lambda}(t)+\mathcal{J}\left(\sigma_{r} B_{r}(t)+\sigma_{\lambda} B_{\lambda}(t)\right),
\end{aligned}
$$

with terminal conditions

$$
B_{r}(u)=0, \quad B_{\lambda}(u)=v, \quad A(u)=0,
$$

and $\mathcal{J}$ given by the formula

$$
J(x)=-\langle a, x\rangle+\int_{\mathbf{R}^{2}}\left(e^{-\langle x, y\rangle}-1+\langle x, y\rangle \mathbf{1}_{\{|y| \leq 1\}}(y)\right) \nu(d y) .
$$

REMARK 5. The unique solution to the above system of ODE has the form:

$$
\begin{aligned}
B_{r}(t, u)= & -\frac{1}{\theta_{r}}\left(e^{-\theta_{r}(u-t)}-1\right), \\
B_{\lambda}(t, u, w, v)= & v e^{-\theta_{\lambda}(u-t)}-\frac{w}{\theta_{\lambda}}\left(e^{-\theta_{\lambda}(u-t)}-1\right), \\
A(t, u, w, v)= & -\kappa_{r}\left(B_{r}(t, u)+(u-t)\right)-\kappa_{\lambda}\left(v-B_{\lambda}(t, u, w, v)+w(u-t)\right) \\
& +\int_{t}^{u} \mathcal{J}\left(\sigma_{r} B_{r}(s, u)+\sigma_{\lambda} B_{\lambda}(s, u, w, v)\right) d s .
\end{aligned}
$$

REMARK 6. Theorem 21 gives us the explicit form of the function $H$ defined by (35) for $k=0$, namely

$$
H(t, u, 0, w)=e^{-B_{r}(t, u) r(t)-B_{\lambda}(t, u, w, 0) \lambda(t)+A(t, u, w, 0)} .
$$

The value of the function $H(\cdot, \cdot, k, \cdot)$ for arbitrary $k \in \mathbf{N}_{+}$can be derived inductively. To do this we use the relation

$$
H(t, u, k, w)=-\frac{1}{k} \frac{\partial H(t, u, k-1, w)}{\partial w},
$$

which follows from the following calculations

$$
\begin{aligned}
\frac{1}{k} \frac{\partial H(t, u, k-1, w)}{\partial w} & =\frac{1}{k} \frac{\partial}{\partial w} \mathbf{E}\left(\frac{\left(\int_{t}^{u} \lambda_{v} d v\right)^{k-1}}{(k-1) !} e^{-\int_{t}^{u}\left(r_{v}+w \lambda_{v}\right) d v} \mid \mathcal{F}_{t}\right) \\
& =\frac{1}{k} \mathbf{E}\left(\frac{\left(\int_{t}^{u} \lambda_{v} d v\right)^{k-1}}{(k-1) !} \frac{\partial}{\partial w} e^{-\int_{t}^{u}\left(r_{v}+w \lambda_{v}\right) d v} \mid \mathcal{F}_{t}\right) \\
& =-\mathbf{E}\left(\frac{\left(\int_{t}^{u} \lambda_{v} d v\right)^{k}}{k !} e^{-\int_{t}^{u}\left(r_{v}+w \lambda_{v}\right) d v} \mid \mathcal{F}_{t}\right) .
\end{aligned}
$$

Changing the order of integration and differentiation is justified by admissibility of the point $(w, v)$. 
The same remark concerns the function defined by (34). For $k=0$ we have

$$
B(t, u, 0, w)=\left.\frac{\partial}{\partial v} e^{-B_{r}(t, u) r(t)-B_{\lambda}(t, u, w, v) \lambda(t)+A(t, u, w, v)}\right|_{v=0}
$$

and, as before, for arbitrary $k \in \mathbf{N}_{+}$we have

$$
B(t, u, k, w)=-\frac{1}{k} \frac{\partial B(t, u, k-1, w)}{\partial w} .
$$

REMARK 7. If an $\mathbb{R}^{2}$-valued $\left(r_{t}, \lambda_{t}\right)$ process is affine (see Duffie et al. [7]), then it is also possible to derive an explicit form of the conditional expectations in (34) and (35).

5. Appendix. In the appendix we present two lemmas used in this paper, and which are also of independent interest, and the proof of Lemma 2 which is probably known, but we couldn't find the proper references. Finally, we quote the known facts from matrix algebra, which we have used in our proofs.

Lemma 22. Let $\mathbb{F}$ be a given filtration, $N$ be a Cox process w.r.t. $\mathbb{F}$ and $\bar{C}$ be a discrete time homogeneous Markov chain independent of $\mathcal{F}_{\infty}$ and with transition matrix $P$. Define the process $\left(C_{t}\right)_{t \geq 0}$ by the formula:

$$
C_{t}=\bar{C}_{N_{t}}
$$

Assume that $\mathbf{P}\left(\bar{C}_{0}=i_{0}\right)=1$ and Assumption 4 is satisfied. Then:

a) For $0 \leq u_{1}<\ldots<u_{n}$

$$
\begin{aligned}
\mathbf{P}\left(C_{u_{1}}\right. & \left.=i_{1}, \ldots, C_{u_{n}}=i_{n} \mid \mathcal{F}_{\infty}\right) \\
& =\prod_{m=1}^{n}\left(\sum_{k_{m}=0}^{\infty}\left[P^{k_{m}}\right]_{i_{m-1}, i_{m}} e^{-\int_{u_{m}-1}^{u_{m}} \lambda_{v} d v} \frac{\left(\int_{u_{m-1}}^{u_{m}} \lambda_{v} d v\right)^{k_{m}}}{k_{m} !}\right) \\
& =\prod_{m=1}^{n}\left[e^{(P-I) \int_{u_{m-1}}^{u_{m}} \lambda_{v} d v}\right]_{i_{m-1}, i_{m}},
\end{aligned}
$$

where we put $u_{0}=0$.

b) For every $n \in \mathbf{N}$ and all sequences $0 \leq u_{1}<\ldots<u_{n} \leq t,\left(i_{1}, \ldots, i_{n}\right) \in \mathcal{K}^{n}$ we have

$$
\mathbf{P}\left(C_{u_{1}}=i_{1}, \ldots, C_{u_{n}}=i_{n} \mid \mathcal{F}_{t}\right)=\mathbf{P}\left(C_{u_{1}}=i_{1}, \ldots, C_{u_{n}}=i_{n} \mid \mathcal{F}_{\infty}\right),
$$

(i.e. the process $\left(C_{t}\right)_{t \geq 0}$ have property ii) from Lemma 2).

Proof. a) Fix $n \in \mathbf{N}$ and sequences $\left(i_{1}, \ldots, i_{n}\right),\left(u_{1}, \ldots u_{n}\right), 0 \leq u_{1}<\ldots<u_{n} \leq t$. Then we have

$$
\begin{aligned}
& \mathbf{P}\left(C_{u_{1}}=i_{1}, C_{u_{2}}=i_{2}, \ldots, C_{u_{n}}=i_{n} \mid \mathcal{F}_{\infty}\right) \\
& =\mathbf{P}\left(\bar{C}_{N_{u_{1}}}=i_{1}, \bar{C}_{N_{u_{2}}}=i_{2}, \ldots, \bar{C}_{N_{u_{n}}}=i_{n} \mid \mathcal{F}_{\infty}\right) \\
& =\mathbf{P}\left(\bar{C}_{N_{u_{1}}-N_{u_{0}}}=i_{1}, \bar{C}_{N_{u_{2}}-N_{u_{1}}+N_{u_{1}}-N_{u_{0}}}=i_{2}, \ldots, \bar{C}_{\sum_{l=1}^{n}\left(N_{u_{l}}-N_{u_{l-1}}\right)}=i_{n} \mid \mathcal{F}_{\infty}\right)
\end{aligned}
$$




$$
\begin{aligned}
& =\sum_{k_{1}, \ldots k_{n}=0}^{\infty} \mathbf{P}\left(\bar{C}_{k_{1}}=i_{1}, \bar{C}_{\sum_{l=1}^{2} k_{l}}=i_{2}, \ldots, \bar{C}_{\sum_{l=1}^{n} k_{l}}=i_{n},\right. \\
& \left.\left.N_{u_{1}}-N_{u_{0}}=k_{1}, N_{u_{2}}-N_{u_{1}}=k_{2}, \ldots, N_{u_{n}}-N_{u_{n-1}}=k_{n}\right) \mid \mathcal{F}_{\infty}\right) \\
& =\sum_{k_{1}, \ldots k_{n}=0}^{\infty} \mathbf{P}\left(\bigcap_{m=1}^{n}\left\{\bar{C}_{\sum_{l=1}^{m} k_{l}}=i_{m}\right\}, \bigcap_{m=1}^{n}\left\{N_{u_{m}}-N_{u_{m-1}}=k_{m}\right\} \mid \mathcal{F}_{\infty}\right) \\
& =\sum_{k_{1}, \ldots k_{n}=0}^{\infty} \mathbf{P}\left(\bigcap_{m=1}^{n}\left\{\bar{C}_{\sum_{l=1}^{m} k_{l}}=i_{m}\right\} \mid \mathcal{F}_{\infty}\right) \mathbf{P}\left(\bigcap_{m=1}^{n}\left\{N_{u_{m}}-N_{u_{m-1}}=k_{m}\right\} \mid \mathcal{F}_{\infty}\right),
\end{aligned}
$$

where the last equality follows from Assumption 4 . Independence of $\left(\bar{C}_{k}\right)_{k \geq 0}$ and $\mathcal{F}_{\infty}$, Markov property of $\left(\bar{C}_{k}\right)_{k \geq 0}$ and $\mathbf{P}\left(\bar{C}_{0}=i_{0}\right)=1$ enable us to write the following formula for the first term in the above product

$$
\begin{aligned}
\mathbf{P}\left(\bigcap_{m=1}^{n}\left\{\bar{C}_{\sum_{l=1}^{m} k_{l}}=i_{m}\right\} \mid \mathcal{F}_{\infty}\right) & =\prod_{m=1}^{n} \mathbf{P}\left(\bar{C}_{\sum_{l=1}^{m} k_{l}}=i_{m} \mid \bar{C}_{\sum_{l=1}^{m-1} k_{l}}=i_{m-1}\right) \\
& =\prod_{m=1}^{n}\left[P^{k_{m}}\right]_{i_{m-1}, i_{m}} .
\end{aligned}
$$

Whereas, by (21),

$$
\mathbf{P}\left(\bigcap_{m=1}^{n}\left\{N_{m}-N_{m-1}=k_{m}\right\} \mid \mathcal{F}_{\infty}\right)=\prod_{m=1}^{n} e^{-\int_{u_{m}-1}^{u_{m}} \lambda_{v} d v} \frac{\left(\int_{u_{m-1}}^{u_{m}} \lambda_{v} d v\right)^{k_{m}}}{k_{m} !},
$$

where we have set $u_{0}=0$ and used the assumption $\mathbf{P}\left(C_{0}=i_{0}\right)=\mathbf{P}\left(N_{0}=0\right)=1$. Therefore

$$
\begin{aligned}
& \mathbf{P}\left(C_{u_{1}}=i_{1}, \ldots, C_{u_{n}}=i_{n} \mid \mathcal{F}_{\infty}\right) \\
& =\sum_{k_{1}, \ldots k_{n}=0}^{\infty} \prod_{m=1}^{n}\left[P^{k_{m}}\right]_{i_{m-1}, i_{m}} e^{-\int_{u_{m-1}}^{u_{m}} \lambda_{v} d v} \frac{\left(\int_{u_{m-1}}^{u_{m}} \lambda_{v} d v\right)^{k_{m}}}{k_{m} !} \\
& =\prod_{m=1}^{n}\left(\sum_{k_{m}=0}^{\infty}\left[P^{k_{m}}\right]_{i_{m-1}, i_{m}} e^{-\int_{u_{m-1}}^{u_{m}} \lambda_{v} d v} \frac{\left(\int_{u_{m-1}}^{u_{m}} \lambda_{v} d v\right)^{k_{m}}}{k_{m} !}\right)
\end{aligned}
$$

which establishes formula (45).

b) The right hand side of (45) is $\mathcal{F}_{u_{n}}$-measurable so, by the chain rule for conditional expectations, formula (46) follows.

LEMma 23. Under the hypothesis of Lemma 22 for any $m \in \mathbf{N}$ and all sequences $t<$ $v_{1}<\ldots<v_{m},\left(j_{1}, \ldots j_{m}\right) \in \mathcal{K}^{m}$, we have

$$
\mathbf{E}\left(\mathbf{1}_{\left\{C_{v_{1}}=j_{1}, \ldots, C_{v_{m}}=j_{m}\right\}} \mid \mathcal{F}_{\infty} \vee \mathcal{F}_{t}^{C}\right)=\mathbf{E}\left(\mathbf{1}_{\left\{C_{v_{1}}=j_{1}, \ldots, C_{v_{m}}=j_{m}\right\}} \mid \mathcal{F}_{\infty} \vee \sigma\left(C_{t}\right)\right) .
$$

Proof. Fix sequences $t<v_{1}<\ldots<v_{m},\left(j_{1}, \ldots, j_{m}\right) \in \mathcal{K}^{m}$. It suffices to show that for every set $D \in \mathcal{F}_{\infty} \vee \mathcal{F}_{t}^{C}$

$$
\mathbf{E}\left(\mathbf{1}_{\left\{C_{v_{1}}=j_{1}, \ldots, C_{v_{m}}=j_{m}\right\}} \mathbf{1}_{D}\right)=\mathbf{E}\left(\mathbf{E}\left(\mathbf{1}_{\left\{C_{v_{1}}=j_{1}, \ldots, C_{v_{m}}=j_{m}\right\}} \mid \mathcal{F}_{\infty} \vee \sigma\left(C_{t}\right)\right) \mathbf{1}_{D}\right) .
$$

Let us denote by $\mathcal{L}$ the class of sets for which equality (47) holds. Obviously, $\mathcal{L}$ constitutes a $\lambda$-system. We will denote by $\mathcal{C}_{t}$ the following class of sets 
$\mathcal{C}_{t}:=\left\{B: B=A \cap H\right.$, where $A \in \mathcal{F}_{\infty}, H=\left\{C_{u_{1}}=i_{1}, \ldots, C_{u_{n}}=i_{n}\right\}$ for some $\left.n \in \mathbf{N}, \max \left\{u_{1}, \ldots u_{n}\right\} \leq t\right\}$.

The class $\mathcal{C}_{t}$ is a $\pi$-system and $\sigma\left(\mathcal{C}_{t}\right)=\mathcal{F}_{\infty} \vee \mathcal{F}_{t}^{C}$. So it is enough to prove (47) for sets belonging to $\mathcal{C}_{t}$, i.e.

$$
\mathbf{E}\left(\mathbf{1}_{\left\{C_{v_{1}}=j_{1}, \ldots, C_{v_{m}}=j_{m}\right\}} \mathbf{1}_{A} \mathbf{1}_{H}\right)=\mathbf{E}\left(\mathbf{1}_{A} \mathbf{1}_{H} \mathbf{E}\left(\mathbf{1}_{\left\{C_{v_{1}}=j_{1}, \ldots, C_{v_{m}}=j_{m}\right\}} \mid \mathcal{F}_{\infty} \vee \sigma\left(C_{t}\right)\right)\right) .
$$

Let $F=\left\{C_{v_{1}}=j_{1}, \ldots, C_{v_{m}}=j_{m}\right\}$. Then, the left hand side of (48) equals

$$
\mathbf{E}\left(\mathbf{1}_{A} \mathbf{E}\left(\mathbf{1}_{F} \mathbf{1}_{H} \mid \mathcal{F}_{\infty}\right)\right)=\sum_{i=1}^{K} \mathbf{E}\left(\mathbf{1}_{A} \mathbf{E}\left(\mathbf{1}_{F} \mathbf{1}_{\left\{C_{t}=i\right\}} \mathbf{1}_{H} \mid \mathcal{F}_{\infty}\right)\right),
$$

and the right hand side of (48), by (3), equals

$$
\begin{aligned}
& \sum_{i=1}^{K} \mathbf{E}\left(\mathbf{1}_{A} \mathbf{1}_{H} \mathbf{1}_{\left\{C_{t}=i\right\}} \frac{\mathbf{E}\left(\mathbf{1}_{\left\{C_{t}=i\right\}} \mathbf{1}_{F} \mid \mathcal{F}_{\infty}\right)}{\mathbf{P}\left(C_{t}=i \mid \mathcal{F}_{\infty}\right)}\right) \\
& =\sum_{i=1}^{K} \mathbf{E}\left(\mathbf{1}_{A} \frac{\mathbf{E}\left(\mathbf{1}_{\left\{C_{t}=i\right\}} \mathbf{1}_{F} \mid \mathcal{F}_{\infty}\right)}{\mathbf{P}\left(C_{t}=i \mid \mathcal{F}_{\infty}\right)} \mathbf{E}\left(\mathbf{1}_{H} \mathbf{1}_{\left\{C_{t}=i\right\}} \mid \mathcal{F}_{\infty}\right)\right) .
\end{aligned}
$$

Therefore, it is enough to prove that

$$
\mathbf{E}\left(\mathbf{1}_{F} \mathbf{1}_{\left\{C_{t}=i\right\}} \mathbf{1}_{H} \mid \mathcal{F}_{\infty}\right)=\frac{\mathbf{E}\left(\mathbf{1}_{\left\{C_{t}=i\right\}} \mathbf{1}_{F} \mid \mathcal{F}_{\infty}\right)}{\mathbf{P}\left(C_{t}=i \mid \mathcal{F}_{\infty}\right)} \mathbf{E}\left(\mathbf{1}_{H} \mathbf{1}_{\left\{C_{t}=i\right\}} \mid \mathcal{F}_{\infty}\right) .
$$

In order to get this equality it is convenient to apply (45) to the sequences $w=$ $\left(w_{0}, \ldots, w_{n+m+1}\right)$ and $c=\left(c_{0}, \ldots, c_{n+m+1}\right)$ given by

$$
w_{k}= \begin{cases}0, & \text { for } k=0, \\ u_{k}, & \text { for } k=1, \ldots, n, \\ t, & \text { for } k=n+1, \\ v_{k-n-1}, & \text { for } k=n+2, \ldots, m+n+1\end{cases}
$$

and

$$
c_{k}= \begin{cases}i_{0}, & \text { for } k=0 \\ i_{k}, & \text { for } k=1, \ldots, n \\ i, & \text { for } k=n+1, \\ j_{k-n-1}, & \text { for } k=n+2, \ldots, m+n+1\end{cases}
$$

We conclude that

$$
\begin{aligned}
\mathbf{E}\left(\mathbf{1}_{F} \mathbf{1}_{\left\{C_{t}=i\right\}} \mathbf{1}_{H} \mid \mathcal{F}_{\infty}\right) & =\prod_{p=1}^{m+n+1}\left(\sum_{k_{p}=0}^{\infty}\left(P^{k_{p}}\right)_{c_{p-1}, c_{p}} e^{-\int_{w_{p-1}}^{w_{p}} \lambda_{v} d v} \frac{\left(\int_{w_{p-1}}^{w_{p}} \lambda_{v} d v\right)^{k_{p}}}{k_{p} !}\right), \\
\frac{\mathbf{E}\left(\mathbf{1}_{\left\{C_{t}=i\right\}} \mathbf{1}_{F} \mid \mathcal{F}_{\infty}\right)}{\mathbf{P}\left(C_{t}=i \mid \mathcal{F}_{\infty}\right)} & =\prod_{l=n+2}^{m+n+1}\left(\sum_{k_{l}=0}^{\infty}\left[P^{k_{l}}\right]_{c_{l-1}, c_{l}} e^{-\int_{w_{l-1}}^{w_{l}} \lambda_{s} d s} \frac{\left(\int_{w_{l-1}}^{w_{l}} \lambda_{s} d s\right)^{k_{l}}}{k_{l} !}\right), \\
\mathbf{E}\left(\mathbf{1}_{H} \mathbf{1}_{\left\{C_{t}=i\right\}} \mid \mathcal{F}_{\infty}\right) & =\prod_{l=1}^{n+1}\left(\sum_{k_{l}=0}^{\infty}\left[P^{k_{l}}\right]_{c_{l-1}, c_{l}} e^{-\int_{w_{l-1}}^{w_{l}} \lambda_{v} d v} \frac{\left(\int_{w_{l-1}}^{w_{l}} \lambda_{v} d v\right)^{k_{l}}}{k_{l} !}\right) .
\end{aligned}
$$

It remains to combine these formulae to obtain (49). 
Now, we give the proof of Lemma 2 since we couldn't find a proper reference.

Proof of Lemma 2. It is a well known fact that i) is equivalent to Assumption 2.

i) $\Rightarrow$ ii $)$. Fix any $n \in \mathbf{N}$ and sequences $u_{1}<\ldots<u_{n} \leq t,\left(i_{1}, \ldots, i_{n}\right) \in \mathcal{K}^{n}$. Denote $A=\left\{C_{u_{1}}=i_{1}, \ldots, C_{u_{n}}=i_{n}\right\}$. For an arbitrary set $F$ from $\mathcal{F}_{\infty}$ we have

$$
\begin{aligned}
\mathbf{E}\left(\mathbf{1}_{F} \mathbf{1}_{A}\right) & =\mathbf{E}\left(\mathbf{E}\left(\mathbf{1}_{F} \mathbf{1}_{A} \mid \mathcal{F}_{t}\right)\right)=\mathbf{E}\left(\mathbf{E}\left(\mathbf{1}_{A} \mid \mathcal{F}_{t}\right) \mathbf{E}\left(\mathbf{1}_{F} \mid \mathcal{F}_{t}\right)\right) \\
& =\mathbf{E}\left(\mathbf{E}\left(\mathbf{1}_{F} \mathbf{E}\left(\mathbf{1}_{A} \mid \mathcal{F}_{t}\right) \mid \mathcal{F}_{t}\right)\right)=\mathbf{E}\left(\mathbf{1}_{F} \mathbf{E}\left(\mathbf{1}_{A} \mid \mathcal{F}_{t}\right)\right),
\end{aligned}
$$

which gives us (2).

ii) $\Rightarrow$ i). Fix $n \in \mathbf{N}$. Let $A \in \mathcal{F}_{t}^{C}$ be a set of the form $A=\left\{C_{u_{1}}=i_{1}, \ldots, C_{u_{n}}=i_{n}\right\}$, where $0 \leq u_{1}<\ldots<u_{n} \leq t,\left(i_{1}, \ldots, i_{n}\right) \in \mathcal{K}^{n}$ and let $F \in \mathcal{F}_{\infty}$ be an arbitrary set. We have to prove that

$$
\mathbf{E}\left(\mathbf{1}_{F} \mathbf{1}_{A} \mid \mathcal{F}_{t}\right)=\mathbf{E}\left(\mathbf{1}_{A} \mid \mathcal{F}_{t}\right) \mathbf{E}\left(\mathbf{1}_{F} \mid \mathcal{F}_{t}\right)
$$

Starting from the right hand side we obtain

$$
\begin{aligned}
\mathbf{E}\left(\mathbf{1}_{A} \mid \mathcal{F}_{t}\right) \mathbf{E}\left(\mathbf{1}_{F} \mid \mathcal{F}_{t}\right) & =\mathbf{E}\left(\mathbf{1}_{F} \mathbf{E}\left(\mathbf{1}_{A} \mid \mathcal{F}_{t}\right) \mid \mathcal{F}_{t}\right)=\mathbf{E}\left(\mathbf{1}_{F} \mathbf{E}\left(\mathbf{1}_{A} \mid \mathcal{F}_{\infty}\right) \mid \mathcal{F}_{t}\right) \\
& =\mathbf{E}\left(\mathbf{E}\left(\mathbf{1}_{F} \mathbf{1}_{A} \mid \mathcal{F}_{\infty}\right) \mid \mathcal{F}_{t}\right)=\mathbf{E}\left(\mathbf{1}_{F} \mathbf{1}_{A} \mid \mathcal{F}_{t}\right)
\end{aligned}
$$

and the result follows since $\mathcal{F}_{t}^{C}$ is generated by the sets of the form $A$.

For convenience of the reader and to fix notation we quote here the known facts from matrix algebra (see [17] or [9]), which we have used in our proofs.

Lemma 24. Let $B=A J A^{-1}$, where $A$ is a nonsingular matrix, $J$ is a Jordan form of $B$, i.e. $J$ is a matrix of the following form

$$
J=\bigoplus_{l=1}^{k} J_{n_{l}}\left(d_{l}\right)
$$

where $J_{n_{l}}\left(d_{l}\right)$ is a Jordan block of dimension $n_{l} \times n_{l}$ associated with eigenvalue $d_{l}$ with multiplicity $n_{l}$. For every $a \in \mathbf{R}$ we have the following formula

$$
e^{a B}=A\left(\bigoplus_{l=1}^{k} e^{a J_{n_{l}}\left(d_{l}\right)}\right) A^{-1}
$$

where $e^{a J_{n_{l}}\left(d_{l}\right)}$ is an upper triangular matrix with general term given by

$$
\left[e^{a J_{n_{l}}\left(d_{l}\right)}\right]_{i, j}= \begin{cases}e^{a d_{l}} \frac{a^{j-i}}{(j-i) !}, & \text { for } j-i \geq 0 \\ 0, & \text { for } j-i<0 .\end{cases}
$$

Moreover, the general term $(i, j)$ of the matrix $e^{a B}$ can be written as

$$
\left[e^{a B}\right]_{i, j}=\sum_{l=1}^{k} \sum_{m=n_{l}^{\prime}+1}^{n_{l}^{\prime}+n_{l}} a_{i, m}\left(\sum_{p=m}^{n_{l}^{\prime}+n_{l}} b_{p, j} e^{a d_{l}} \frac{a^{p-m}}{(p-m) !}\right),
$$

where $n_{1}^{\prime}=0, n_{l}^{\prime}=n_{l-1}^{\prime}+n_{l}$ for $l=1, \ldots, k$ and $b_{p, j}:=\left[A^{-1}\right]_{p, j}$. 


\section{References}

[1] V. V. Acharya, S. R. Das, and R. K. Sundaram, Pricing credit derivatives with rating transitions, Financial Analysts Journal 58 (2002), 28-44.

[2] D. Applebaum, Lévy Processes and Stochastic Calculus, Cambridge University Press, 2004.

[3] T. Bielecki, S. Crepey, M. Jeanblanc, and M. Rutkowski, Valuation of basket credit derivatives in the credit migrations environment, in: J. Birge and V. Linetsky (eds.), Handbook on Financial Engineering, Elsevier, 2007.

[4] T. Bielecki and M. Rutkowski, Multiple ratings model of defaultable term structure, Mathematical Finance 10 (2000), 125-139.

[5] T. Bielecki and M. Rutkowski, Credit Risk: Modeling, Valuation and Hedging, Springer Finance, Springer-Verlag, Berlin, 2001.

[6] T. Bielecki and M. Rutkowski, Modeling of the defaultable term structure: Conditionally Markov approach, IEEE Transactions on Automatic Control 49 (2004), 361-373.

[7] S. R. Das and P. Tufano, Pricing credit-sensitive debt when interest rates, credit ratings and credit spreads are stochastic, Journal of Financial Engineering 5 (1996), 161-198.

[8] D. Duffie, D. Filipović and W. Schachermayer, Affine processes and applications in finance, Annals of Applied Probability 13 (2003), 984-1053.

[9] E. Eberlein and F. Özkan, The defaultable Lévy term structure: Ratings and restructuring, Mathematical Finance 13 (2003), 277-300.

[10] G. H. Golub and C. Van Loan, Matrix Computations, 3rd ed., The Johns Hopkins University Press, 1996.

[11] Ch. M. Grinstead and J. L. Snell, Introduction to Probability, 2nd ed., American Mathematical Society, 1997.

[12] T. R. Hurd and A. Kuznetsov, Fast CDO computations in the affine Markov chain model, working paper, 2006.

[13] T. R. Hurd and A. Kuznetsov, Affine Markov chain model of multifirm credit migration, Journal of Credit Risk 3 (2007), 3-29.

[14] J. Jakubowski, M. Niewęgłowski, and J. Zabczyk, Lévy modelling of defaultable bonds, Inst. of Math., Polish Acad. of Sciences Preprint 658 (2005), 22 pp.

[15] J. Jakubowski and J. Zabczyk, Exponential moments for HJM models with jumps, Finance and Stochastics 11 (2007), 429-445.

[16] R. A. Jarrow, D. Lando, and T. S. Turnbull, A Markov model for the term structure of credit risk spreads, The Review of Financial Studies 10 (1997), 481-523.

[17] D. Lando, On Cox processes and credit-risky securities, Review of Derivatives Research 2 (1998), 99-120.

[18] C. Moler and C. Van Loan, Nineteen dubious ways to compute the exponential of a matrix, twenty-five years later, SIAM Review 45 (2003), 3-49.

[19] M. Niewęgłowski, Wybrane aspekty ryzyka obligacji modelowanych procesami Lévy'ego, PHD thesis, Warsaw University of Technology, 2007.

[20] T. Schmidt, An infinite factor model for credit risk, International Journal of Theoretical and Applied Finance 9 (2006), 43-68. 